\title{
Boosting LPMO-driven lignocellulose degradation by polyphenol oxidase-activated lignin building blocks
}

\author{
Matthias Frommhagen', Sumanth Kumar Mutte ${ }^{2}$, Adrie H. Westphal ${ }^{2}$, Martijn J. Koetsier ${ }^{3}$, Sandra W. A. Hinz ${ }^{3}$, \\ Jaap Visser ${ }^{4}$, Jean-Paul Vincken ${ }^{1}$, Dolf Weijers ${ }^{2}$, Willem J. H. van Berkel ${ }^{2}$, Harry Gruppen ${ }^{1}$ and Mirjam A. Kabel ${ }^{1 *}$
}

\begin{abstract}
Background: Many fungi boost the deconstruction of lignocellulosic plant biomass via oxidation using lytic polysaccharide monooxygenases (LPMOs). The application of LPMOs is expected to contribute to ecologically friendly conversion of biomass into fuels and chemicals. Moreover, applications of LPMO-modified cellulose-based products may be envisaged within the food or material industry.

Results: Here, we show an up to 75-fold improvement in LPMO-driven cellulose degradation using polyphenol oxidase-activated lignin building blocks. This concerted enzymatic process involves the initial conversion of monophenols into diphenols by the polyphenol oxidase MtPPO7 from Myceliophthora thermophila C1 and the subsequent oxidation of cellulose by MtLPMO9B. Interestingly, MtPPO7 shows preference towards lignin-derived methoxylated monophenols. Sequence analysis of genomes of 336 Ascomycota and 208 Basidiomycota reveals a high correlation between MtPPO7 and AA9 LPMO genes.

Conclusions: The activity towards methoxylated phenolic compounds distinguishes MtPPO7 from well-known PPOs, such as tyrosinases, and ensures that MtPPO7 is an excellent redox partner of LPMOs. The correlation between MtPPO7 and AA9 LPMO genes is indicative for the importance of the coupled action of different monooxygenases in the concerted degradation of lignocellulosic biomass. These results will contribute to a better understanding in both lignin deconstruction and enzymatic lignocellulose oxidation and potentially improve the exploration of eco-friendly routes for biomass utilization in a circular economy.
\end{abstract}

Keywords: LPMO, Myceliophthora thermophila C1, Phenols, Tyrosinase, Catechol oxidase, Polyphenol oxidase, PPO, Agaricus bisporus, Ascomycota, Basidiomycota, Lignocellulose

\section{Background}

Fungal carbohydrate converting enzymes are considered important for eco-friendly application in plant biomass degradation. The resulting carbohydrates are sources for the production of biochemicals or biofuels and new enzymatically modified cellulose-based products are envisaged for the future.

Next to carbohydrates, phenolic compounds are also major components of lignocellulosic plant biomass.

\footnotetext{
*Correspondence: mirjam.kabel@wur.nl

1 Laboratory of Food Chemistry, Wageningen University \& Research,

Bornse Weilanden 9, 6708 WG Wageningen, The Netherlands

Full list of author information is available at the end of the article
}

Phenolic compounds are present either as free molecules or in conjugated form as part of lignin or bound to carbohydrates. Lignin is one of the major constituents of (secondary) plant cell walls, together with the polysaccharides cellulose and hemicellulose. Lignin is composed of the three aromatic monolignol units: coniferyl, sinapyl, and para-coumaryl alcohol. Cellulose consists of $\beta$-(1 $\rightarrow 4)$-linked glucosyl chains that interact with each other via hydrogen bonds and van der Waals forces, which results in the formation of crystalline cellulose fibrils. Unlike cellulose, hemicellulose is a heteropolymer varying in its monosaccharide composition and linkages between the monosaccharides. Examples are xylan, 
mannan, or $\beta$ - $(1 \rightarrow 3,1 \rightarrow 4)$-linked $\beta$-glucan. Hemicellulose interacts with lignin through ester and ether linkages, thereby forming a network that embeds the cellulose microfibrils $[1,2]$.

Recent studies focused on the function of lytic polysaccharide monooxygenases (LPMOs) have confirmed that these enzymes drive the oxidative degradation of cellulose, and they are considered important for the enzymatic degradation of plant biomass [3]. LPMOs are classified based on their sequence in the Carbohydrate Active enzyme (CAZy; [4]) database as auxiliary activity (AA) families AA9, AA10, AA11, and AA13. In brief, LPMOs have been reported to oxidize $\beta-(1 \rightarrow 4)$-linked glucan chains at either the $\mathrm{C} 1$ - or $\mathrm{C} 4$-carbon position or both, resulting in the cleavage of glucan chains $[3,5,6]$. Other LPMOs of these AA families have been described to oxidize the $(1 \rightarrow 4)$-linkage of chitin, xylan, hemicellulose, such as xyloglucan and glucomannan, soluble cellodextrins, and starch [7-11].

In order to oxidize polysaccharides, LPMOs demand electrons to activate molecular oxygen in their coppercontaining active site $[3,5,6]$. The electrons can be donated by reducing agents, like low molecular weight compounds (ascorbic acid, gallic acid) or the macromolecule lignin [12-14]. Other ways of providing LPMOs with electrons have been reported, such as flavocytochrome-dependent cellobiose dehydrogenases (CDHs), light-induced pigments, light-driven chemical oxidation of water, or diphenol-regenerating GMC (glucose-methanol-choline-oxidase/dehydrogenase)-oxidoreductases $[5,12,15,16]$. The mechanistic understanding of electron donation systems is highly relevant in order to enable optimization of LPMO activity and, thereby, plant biomass degradation.

Phenolic compounds, including small molecular weight compounds that serve as lignin building blocks and lignin, are intrinsically present in plant biomass and are natural electron donors for LPMO activity. However, monophenols are not optimal electron donors for LPMO activity, because of their relatively high redox potential [12]. Compounds with a 1,2-benzenediol or a 1,2,3-benzenetriol moiety have, compared to monophenols, a lower redox potential [13]. Their low redox potential enables them to reduce the copper ion in the active site of LPMOs and enhance the LPMO activity [12].

The enzymatic oxidation of phenolic compounds is a well-known reaction in many natural environments. For example, it causes browning of food products, contributes to taste in tea fermentation, and plays a role in plant biomass decomposition [17-19]. These oxidative systems involve the activity of laccases (EC.1.10.3.2), peroxidases (EC.1.11.1), tyrosinases (EC.1.14.18.1), and catechol oxidases (EC 1.10.3.1) [18-20]. In particular tyrosinases, also often referred to as polyphenol oxidases, are of interest due to their ability to hydroxylate phenolic compounds [21]. This so-called monophenolase activity typically involves the ortho-hydroxylation of monophenols into ortho-diphenols, compounds that comprise a 1,2-benzenediol moiety. Tyrosinases also exhibit diphenolase activity, which is characterized by the oxidation of these ortho-diphenols into ortho-quinones [22]. With respect to biomass degradation, the diphenolase activity of polyphenol oxidases is not conductive to polysaccharides oxidation since ortho-quinones cannot be utilized by LPMOs [12]. Based on their monophenolase activity, tyrosinases have been shown to use non-methoxylated monophenols as substrates, rather than using methoxylated monophenols, that are the predominant structural units of lignin [23].

In this study, we investigated if polyphenol oxidases, in particular tyrosinases, can enhance LPMO activity. Therefore, the tyrosinase MtPPO7 from Myceliophthora thermophila $\mathrm{C} 1$ was used, which showed activity towards phenolic compounds and was obtained from DuPont Industrial Biosciences. In addition, we used the commercially available tyrosinase $A b P P O$ from the white button mushroom Agaricus bisporus. Both tyrosinases were each incubated with the previously described $M t$ LPMO9B in the presence of various plant phenolic compounds and cellulose $[13,24]$. We found that $M t \mathrm{PPO} 7$ is highly active towards methoxylated monophenols, including monomeric lignin building blocks. This activity can strongly boost the release of oxidized gluco-oligosaccharides as catalyzed by $M t$ LPMO9B and thereby the degradation of cellulose. In addition, we found a strong correlation between genes encoding MtPPO like proteins and AA9 LPMOs in fungal genomes of 336 Ascomycota and 208 Basidiomycota.

\section{Results \\ MtPPO7 addition improves cellulose oxidation by MtLPMO9B}

Our previous results have shown that reducing agents with a 1,2-benzenediol or 1,2,3-benzenetriol moiety gave the highest release of non-oxidized and C1-oxidized gluco-oligosaccharides from regenerated amorphous cellulose (RAC) incubated with three $M t$ LPMOs compared to the incubation with compounds comprising only a single hydroxyl group [13]. Hence, in the current research we hypothesized that $M t \mathrm{LPMO}$ activity can benefit from enzymes which have hydroxylating capacity, such as polyphenol oxidases (Fig. 1) [21]. We choose MtPPO7, which originates like the MtLPMO9B employed here, from the thermophilic filamentous fungus Myceliophthora thermophila $\mathrm{C} 1$. As a reference, the well-studied tyrosinase AbPPO from the edible mushroom Agaricus bisporus was used [19, 25-28]. 


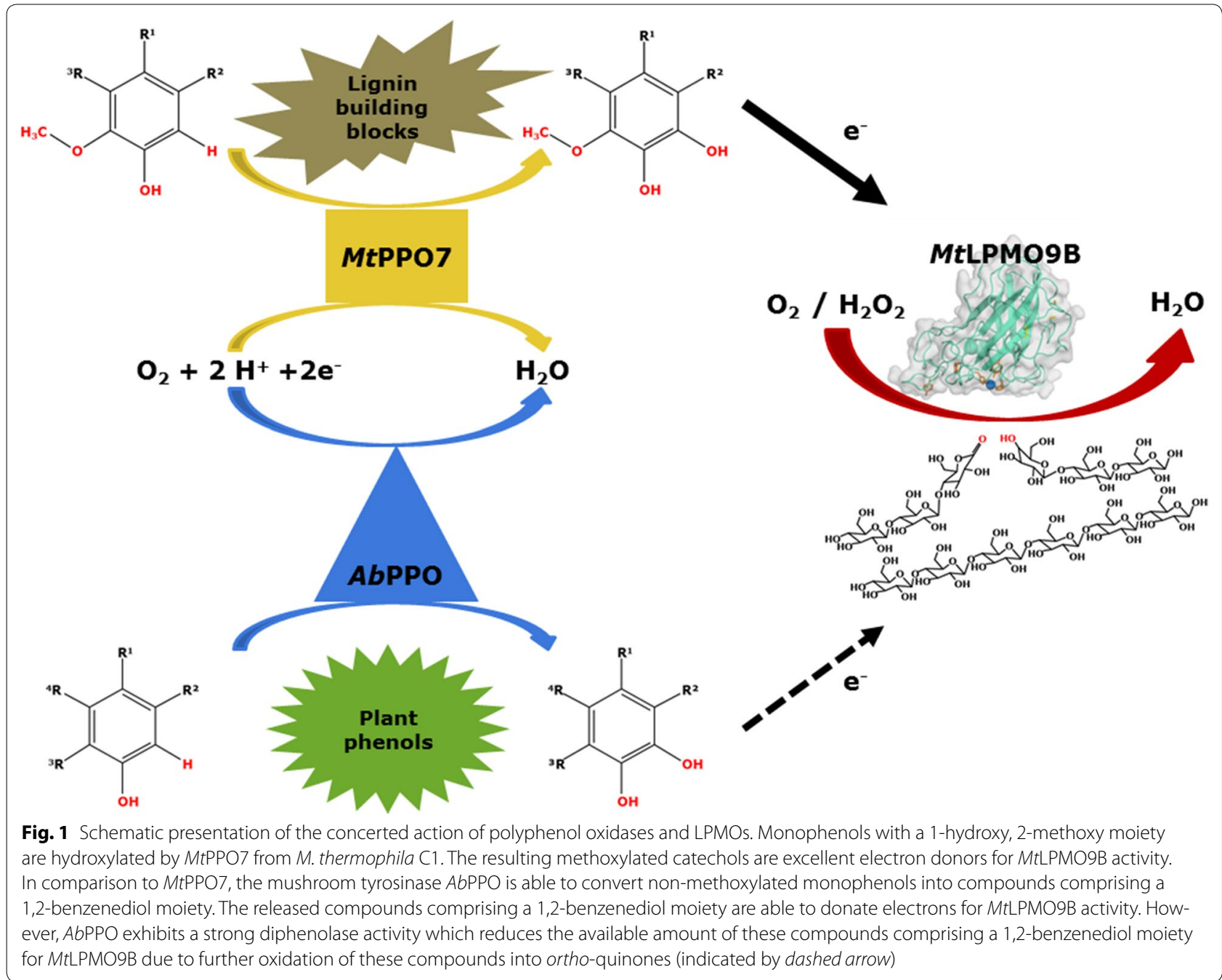

The activity of $M t$ LPMO9B towards RAC, with and without addition of $M t \mathrm{PPO}$, was determined using 21 phenolic compounds as potential electron donors. Many of these phenolic compounds are methoxylated as these are the predominant structural units of lignin. Results are presented in Table 1 and Fig. 2. The 21 different phenolic compounds used were classified into three groups and further divided into subgroups, as previously described [13]. In short, group I represents compounds comprising one hydroxyl group, group II compounds comprising a 1,2-benzenediol moiety, and group III compounds comprising a 1,2,3-benzenetriol moiety (Fig. 2; Table 1).

As expected, from our previous research [13], monophenols (group Ia) are less efficient electron donors for $M t \mathrm{LPMO} 9 \mathrm{~B}$, with the exception of 4-hydroxybenzoic acid (no. 1). The low MtLPMO9B activity towards RAC resulted in a very small amount of released non-oxidized and C1-oxidized gluco-oligosaccharides (Fig. 2; Table 1; Additional file 1: Figure S1). The addition of MtPPO7 to RAC incubated with $M t$ LPMO9B did not increase the electron donor capacities of this group Ia compounds, except for para-coumaric acid (no. 3). In contrast, the addition of $M t \mathrm{PPO} 7$ to RAC incubated with $M t \mathrm{LPMO} 9 \mathrm{~B}$ in the presence of methoxylated monophenols (group Ib, compounds with a 1-hydroxy, 2-methoxy moiety) led in all cases to a high increase (up to \pm 75 times, no. 5 ) in the release of non-oxidized and $\mathrm{C} 1$-oxidized gluco-oligosaccharides (Fig. 2; Additional file 1: Figure S1). Similarly, the oxidative cleavage of RAC incubated with $M t \mathrm{LP}$ MO9B increased, when $M t \mathrm{PPO} 7$ was added to group Ic compounds, which comprise a 1-hydroxy-2,6-dimethoxy moiety. For group IIa, IIb, and III phenolic compounds, the addition of $M t \mathrm{PPO} 7$ to incubations of $\mathrm{RAC}$ with $M t$ LPMO9B led to a moderate increase of non-oxidized 
Table 1 RAC incubated with MtLPMO9B only and with AbPPO or MtPPO7 addition in the presence of 21 reducing agents

\begin{tabular}{|c|c|c|c|c|c|c|}
\hline Gr. & Sub-group & No. & Reducing agent & $\begin{array}{l}\text { Activity [\%] } \\
\text { MtLPMO9B }\end{array}$ & $\begin{array}{l}\text { Activity [\%] } \\
\text { MtLPMO9B + AbPPO }\end{array}$ & $\begin{array}{l}\text { Activity [\%] } \\
\text { MtLPMO9B + MtPPO7 }\end{array}$ \\
\hline \multirow[t]{4}{*}{ । } & a & 1 & 4-Hydroxybenzoic acid & 100 & 119 & 90 \\
\hline & & 2 & Ortho-cresol & 100 & 778 & 2 \\
\hline & & 3 & Para-coumaric acid & 100 & 9938 & 2851 \\
\hline & & 4 & Phenol & $0^{c}$ & $>0^{c}$ & $0^{c}$ \\
\hline \multirow[t]{8}{*}{ । } & $b$ & 5 & 3-Hydroxy-4-methoxycinnamic acid & 100 & 151 & 7558 \\
\hline & & 6 & 4-Hydroxy-3-methoxyphenylacetone & 100 & 117 & 131 \\
\hline & & 7 & Coniferyl aldehyde & 100 & 128 & 562 \\
\hline & & 8 & Ferulic acid & 100 & 107 & 231 \\
\hline & & 9 & Guaiacol & 100 & 461 & 5143 \\
\hline & & 10 & Hesperidin & 100 & 56 & 771 \\
\hline & & 11 & Homovanillic acid & 100 & 156 & 580 \\
\hline & & 12 & Vanillic acid & 100 & 196 & 495 \\
\hline \multirow[t]{3}{*}{ । } & c & 13 & 4-Allyl-2,4-methoxyphenol & 100 & 89 & 108 \\
\hline & & 14 & Sinapic acid & 100 & 110 & 195 \\
\hline & & 15 & Syringic acid & 100 & 107 & 142 \\
\hline \multirow[t]{3}{*}{ ॥ } & a & 16 & 3,4-Dihydroxybenzoic acid & 100 & 99 & 139 \\
\hline & & 17 & 3-Methylcatechol & 100 & 58 & 104 \\
\hline & & 18 & caffeic acid & 100 & 55 & 122 \\
\hline \multirow[t]{2}{*}{ ॥ } & $b$ & 19 & 3,4-Dihydroxy-5-methoxybenzoic acid & 100 & 99 & 22 \\
\hline & & 20 & 3,4-Dihydroxy-5-methoxycinnamic acid & 100 & 99 & 116 \\
\hline III & & 21 & Gallic acid & 100 & 105 & 124 \\
\hline
\end{tabular}

Division based on functional groups of reducing agents (Fig. 2)

a Total release of non-oxidized and C1-oxidized gluco-oligosaccharides from RAC incubated with MtLPMO9B set to 100\%

b Increased (italics $\geq 50 \%$ increase) or decreased percentage of released non-oxidized and C1-oxidized gluco-oligosaccharides from RAC incubated with MtLPMO9B with addition of either AbPPO or MtPPO7 compared to the release of non-oxidized and C1-oxidized gluco-oligosaccharides from RAC incubated with MtLPMO9B alone. Sum of areas of released non-oxidized and C1-oxidized gluco-oligosaccharides are shown in Fig. 2. See "Methods" for more information

c No reference due to absent activity of MtLPMO9B towards RAC in the presence of phenol (Fig. 2)

and C1-oxidized gluco-oligosaccharides (4-39\%), with one exception (no. 19) (Fig. 2; Table 1).

Incubations performed with $A b P P O$ showed deviating results from corresponding experiments with $M t \mathrm{PPO}$. First, unlike $M t \mathrm{PPO}$, addition of $A b P P O$ to non-methoxylated monophenols (group Ia) resulted in increased levels of non-oxidized and C1-oxidized oligosaccharides during the incubation of RAC with $M t$ LPMO9B. This different activity was expected on the basis of the known hydroxylating capacity of $A b \mathrm{PPO}$ towards non-methoxylated monophenolic compounds. Especially, the addition of $A b \mathrm{PPO}$ in the presence of para-coumaric acid (no. 3) increased $M t$ LPMO9B-catalyzed degradation of RAC compared to the incubation without $A b P P O$ (Fig. 2; Table 1). In contrast to MtPPO7, AbPPO addition to group Ib, Ic, II, and III compounds improved the $M t \mathrm{LP}-$ MO9B-catalyzed RAC degradation relatively moderate (5-361\%, Table 1) or even decreased the RAC degradation by $1-44 \%$ (Fig. 2; Table 1 ).
MtLPMO9B-mediated cellulose oxidation in the presence of MtPPO7 using time-dependent incubations

The ability of MtPPO7 to convert methoxylated phenolic compounds and to increase the oxidative degradation of RAC by $M t$ LPMO9B was further investigated using time-dependent incubations $(24 \mathrm{~h})$. These incubations were performed in the presence of two methoxylated monophenols (guaiacol and ferulic acid, group Ib), as well as in the presence of a non-methoxylated ortho-diphenol (3-methylcatechol, group IIa) (Fig. 3; Additional file 2: Figure S2). After $4 \mathrm{~h}$ of incubation, a steady MtPPO7-induced oxidation of guaiacol (no. 9) was observed (Additional file 3: Figure S3). At the same time, the amounts of released non-oxidized and $\mathrm{C} 1$-oxidized gluco-oligosaccharides by $M t$ LPMO9B from RAC also increased steadily (Fig. 3c). A similar trend for the release of non-oxidized and $\mathrm{C} 1$-oxidized gluco-oligosaccharides was shown for the incubation with ferulic acid (no. 8, Additional file 2: Figure S2). MtPPO7 showed a 


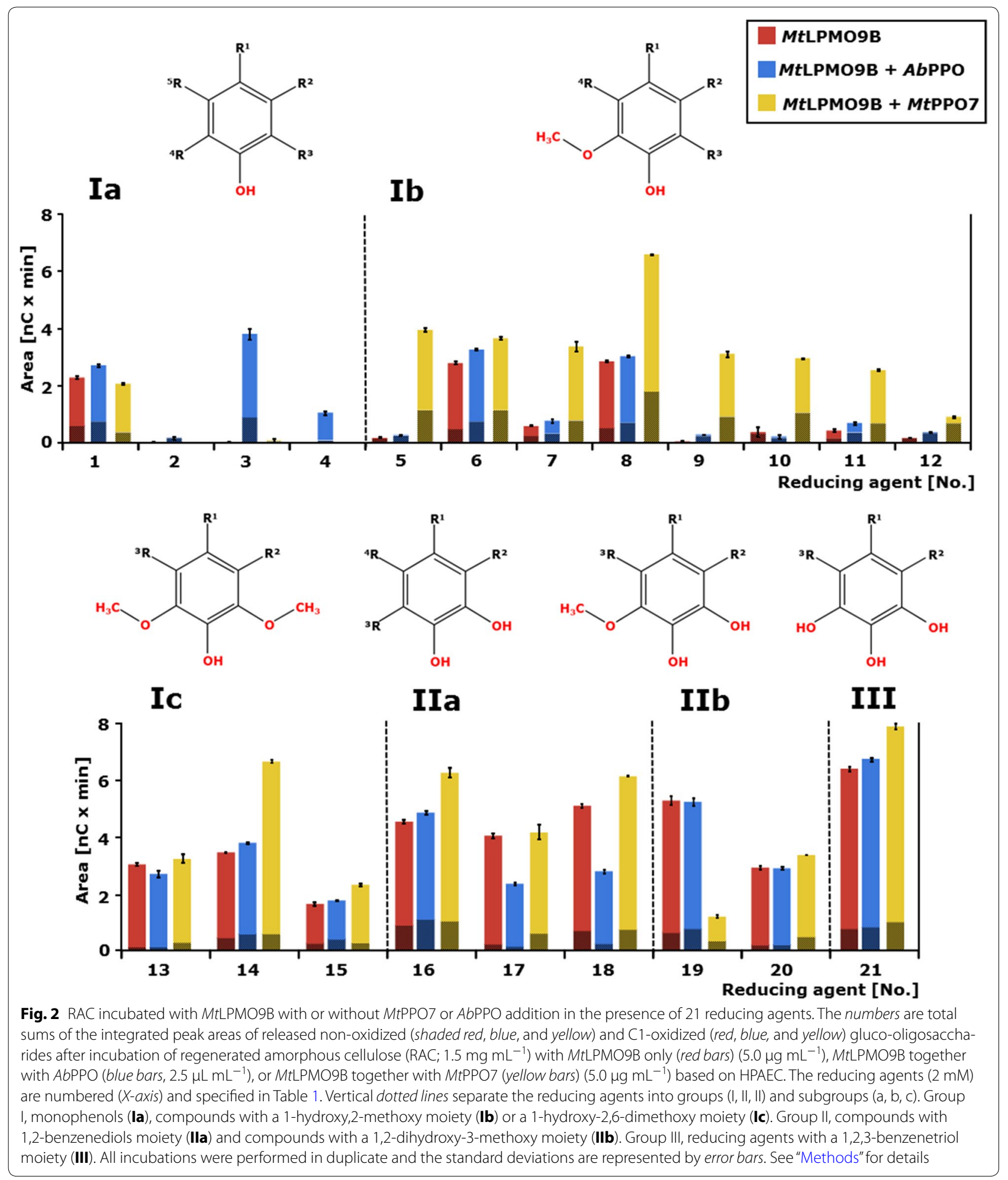

relatively low efficiency towards 3-methylcatechol (no. 17) compared to guaiacol (Table 1; Additional file 3: Figure S3). As reported above, no significant increase of
$M t$ LPMO9B-released non-oxidized and C1-oxidized gluco-oligosaccharides from RAC was observed when comparing incubations with and without MtPPO7 


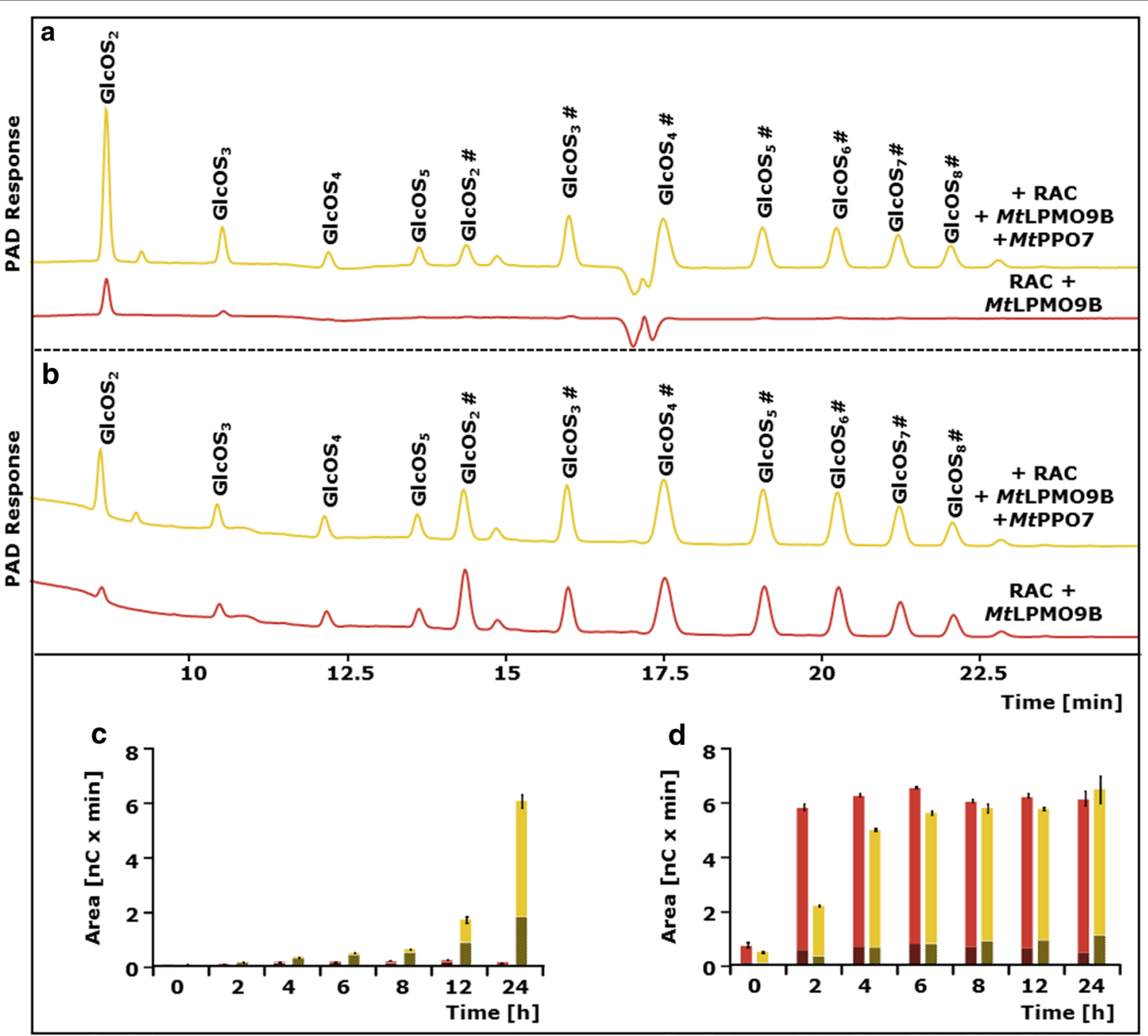

Fig. 3 Activity of MtLPMO9B towards amorphous cellulose in the presence and absence of MtPPO7. HPAEC elution pattern of regenerated amorphous cellulose (RAC; $1.5 \mathrm{mg} \mathrm{mL}^{-1}$ ) incubated with MtLPMO9B $\left(5.0 \mu \mathrm{g} \mathrm{mL}{ }^{-1}\right.$ ) with (yellow) and without (red) MtPPO7 $\left(5.0 \mu \mathrm{g} \mathrm{mL} \mathrm{m}^{-1}\right)$ addition in the presence of a guaiacol (no. 9 specified in Table 1,2 mM) and b 3-methylcatechol (no. 17 specified in Table 1, 2 mM) after $24 \mathrm{~h}$. The incubation of RAC with MtLPMO9B results in the formation of non-oxidized gluco-oligosaccharides $\left(G_{1} \mathrm{COS}_{n}\right)$ and C1-oxidized gluco-oligosaccharides $\left(\mathrm{GlCOS}_{n}^{\#}\right)$. Based on HPAEC, integrated peak areas are shown as the total sum of released non-oxidized (shaded red and yellow) and C1-oxidized (red and yellow) gluco-oligosaccharides after incubation of RAC $\left(1.5 \mathrm{mg} \mathrm{mL}^{-1}\right.$ ) with MtLPMO9B only (red bars, $5.0 \mathrm{\mu g} \mathrm{mL}^{-1}$ ) and MtLPMO9B together with MtPPO7 (yellow bars, $5.0 \mathrm{\mu g} \mathrm{mL}^{-1}$ ) in the presence of $\mathbf{c}$ guaiacol and $\mathbf{d}$ 3-methylcatechol. All incubations were performed in duplicate, and the standard deviations are presented as error bars. See "Methods" for data analysis and details

(Fig. 3d). In presence of $M t$ PPO7 though, the initial rate $(0-6 \mathrm{~h})$ of released non-oxidized and $\mathrm{C} 1$-oxidized glucooligosaccharides was lower compared to the incubation without MtPPO7 (Fig. 3d).

\section{Activity of MtPPO7 towards methoxylated phenolic compounds}

The most striking observation from the experiments described above is the conversion of methoxylated phenolic compounds by MtPPO7 into products, which enhance the oxidative degradation of cellulose by $M t L P-$ MO9B. The fate of these phenolic compounds upon
$M t$ PPO7 incubation was further studied in the absence of $M t$ LPMO9B using UHPLC-UV-MS ${ }^{\mathrm{n}}$.

Based on UV measurements, non-methoxylated phenolic compounds of group Ia, group IIa, and group III remained constant in concentration during $M t P P O 7$ incubation, showing that $M t \mathrm{PPO} 7$ has a low activity towards compounds comprising a 1,2-benzenediol moiety (Fig. 4; Additional file 4: Table S1). Different from MtPPO7, in previous research $A b \mathrm{PPO}$ has been reported to be highly active towards these types compounds [19, 24, 25].

MtPPO7 was active towards all eight methoxylated monophenols (group Ib) tested. Based on UV, the 


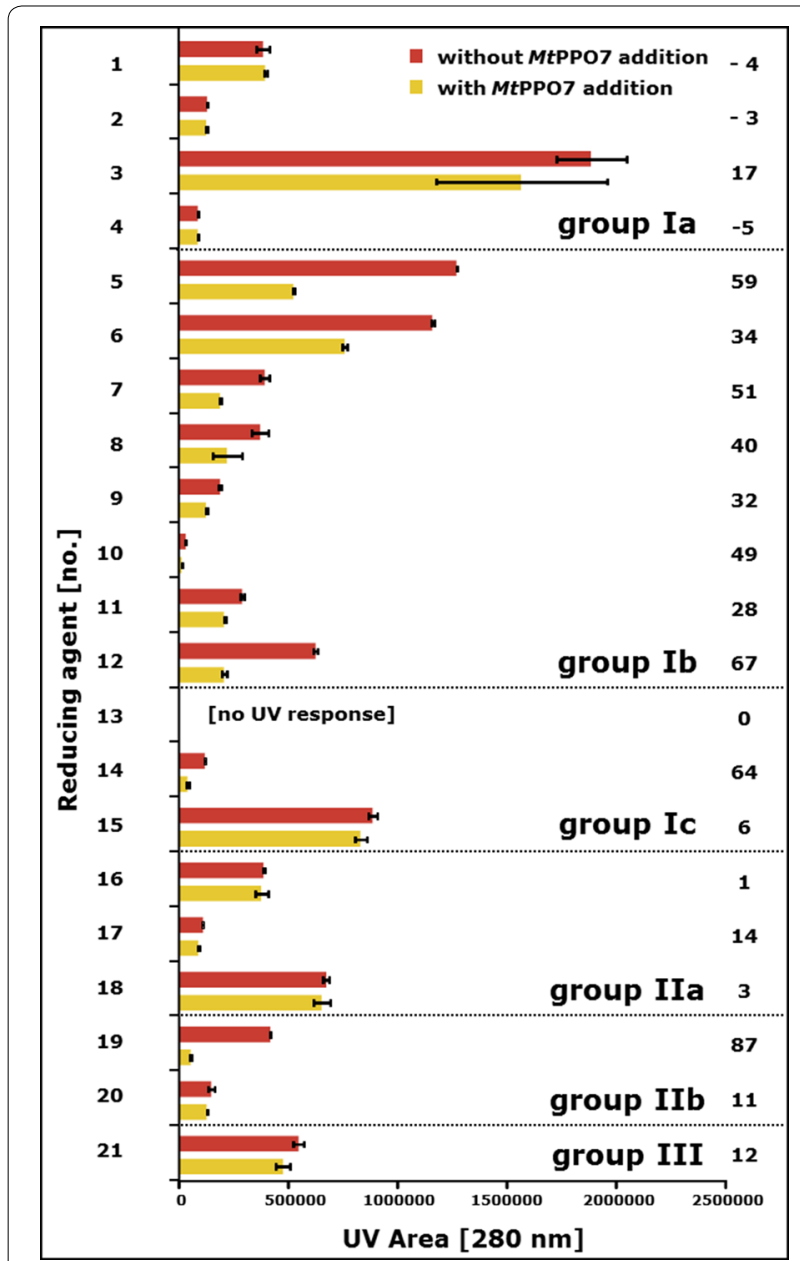

Fig. 4 UV response areas of 21 reducing agents incubated in the presence and absence of MtPPO7. The total sum is shown of integrated peak areas of 21 reducing agents $(2 \mathrm{mM})$ with (yellow bar) and without (red bar) addition of MtPPO7 $\left(5.0 \mathrm{\mu g} \mathrm{mL}^{-1}\right)$. The reducing agents are numbered (bold on the left) and specified in Table 1. Samples were incubated in a $50 \mathrm{mM}$ potassium phosphate buffer $(\mathrm{pH}=6.0)$ containing $2.5 \mu \mathrm{M}$ copper(II)-chloride for $24 \mathrm{~h}$ at $50^{\circ} \mathrm{C}$ and measured by UHPLC-UV ( $280 \mathrm{~nm})$. Bold numbers on the right reducing agents conversion (\%) by MtPPO7, which is based on the difference of integrated peak areas (UV $280 \mathrm{~nm}$ ) of the reducing agents incubated with MtPPO7 compared to the incubation of reducing agents only. All incubations were performed in duplicate. See "Methods" for details

concentrations of group Ib compounds decreased during $M t \mathrm{PPO} 7$ incubation between 28 and 67\% compared to the same compounds incubated without $\mathrm{MtPPO} 7$ (Fig. 4). Three types of $M t \mathrm{PPO} 7$ reactions were observed based on mass differences between substrate and products formed (Additional file 4: Table S1): (A) hydroxylation $(m / z+16$; e.g., no. 5 and 7$)$, (B) decarboxylation $(m / z-44$, e.g., no. 8), and (C) demethylation $(m / z-14$, e.g., no. 11). Especially the hydroxylation of methoxylated phenolic compounds is a key reaction, since the products formed comprise a second hydroxyl group. The formed ortho-diphenols are known to be efficient electron donors for LPMOs. Decarboxylation and demethylation occurred either in the presence of $M t \mathrm{PPO} 7$ or as a result of polymerization reactions in both the presence and absence of MtPPO7. Several group Ib compounds spontaneously formed dimers in the absence of $M t \mathrm{PPO} 7$ (no. 8 or no. 11). These dimers were almost absent when group Ib compounds were incubated with MtPPO7 (Additional file 4: Table S1). Similar to group Ib compounds, the concentrations of compounds comprising a 1-hydroxyl-2,6-dimethoxy moiety (group Ic) incubated with $M t \mathrm{PPO} 7$ decreased, based on UV, between $6 \%$ and $64 \%$ compared to the incubation without MtPPO7 (Fig. 4; Additional file 4: Table S1). Again, masses indicating decarboxylation $(-44 \mathrm{Da})$ and demethylation $(-14 \mathrm{Da})$ reactions were formed. $M t \mathrm{PPO} 7$ was also able to convert group IIb compounds (up to $87 \%$ substrate conversion, no. 20) that comprise a 1,2-dihydroxy-3-methoxy moiety (Fig. 4). The reactions observed were decarboxylation $(m / z-44$, e.g., no. 19) and dimerization (e.g., no. 20) of group IIb compounds based on masses formed (Additional file 4: Table S1).

In general, most phenolic compounds (such as no. 17 and no. 21) that were incubated with $M t \mathrm{PPO} 7$ formed insoluble complexes, which likely result from polymerization reactions of ortho-quinones formed. These insoluble complexes were not determined by UHPLC-UV-MS ${ }^{\mathrm{n}}$. The complexation of ortho-quinones resulted, obviously, in a decrease of the UV signal of the substrates (Fig. 4; Additional file 5: Figure S4).

\section{Conversion of guaiacol and 3-methylcatechol by MtPPO7}

For a better discrimination between monophenolase and diphenolase activity, MtPPO7 conversion of guaiacol and 3-methylcatechol was monitored over a period of $24 \mathrm{~h}$ by UHPLC-UV-MS ${ }^{\mathrm{n}}$. The conversion of guaiacol by $M t \mathrm{PPO} 7$ resulted in the initial formation of 3-methoxycatechol (Additional file 4: Table S1; Additional file 5: Figure S4, Additional file 6: Figure S5). Further reactions resulted in the formation of brown pigments indicating that 3-methoxycatechol was oxidized into ortho-quinones, which are likely to polymerize and form insoluble complexes. Other masses determined by UHPLC-UV$\mathrm{MS}^{\mathrm{n}}$ indicated the polymerization of guaiacol and compounds originating from the oxidation of guaiacol by $M t$ PPO7 into trimers $(\mathrm{m} / z$ 399, 401, 415). Based on the masses detected (Additional file 4: Table S1; Additional file 5: Figure S4), a scheme is presented of possible reaction pathways of guaiacol occurring during $M t \mathrm{PPO} 7$ incubation (Additional file 6: Figure S5).

The oxidation of guaiacol by $M t \mathrm{PPO} 7$ mainly occurred between 4 and $8 \mathrm{~h}$ of incubation, whereas 
3-methylcatechol showed to be oxidized by $M t \mathrm{PPO} 7$ within the first $2 \mathrm{~h}$. During the incubation of 3-methylcatechol with MtPPO7 pink pigments are formed, which precipitate after sample centrifugation. Based on $\mathrm{MS}^{\mathrm{n}}$, masses of 3-methylcatechol $(\mathrm{m} / z$ 123) and masses indicating the dimerization of 3-methylcatechol $(\mathrm{m} / \mathrm{z}$ 245) were detected upon incubation of both 3-methylcatechol with $M t \mathrm{PPO} 7$ and 3-methylcatechol alone (Additional file 4: Table S1). No detectable amounts of new products were formed during the incubation of 3-methylcatechol with MtPPO7 compared to the incubation of 3-methylcatechol only (Additional file 4: Table S1).

\section{Structural model of $\mathrm{MtPPO}$}

A structural model of $M t \mathrm{PPO} 7$ was generated based on the available structure of a catechol oxidase from Aspergillus oryzae (AoCO4, Protein Data Bank entry: 4j3p) (Fig. 5). MtPPO7 and AoCO4 share 38\% amino acid sequence identity. The structural model of $M t \mathrm{PPO} 7$ shows a four-helix bundle fold with the presence of six conserved histidines coordinating the two copper ions in the active site, which is typical for PPO-like tyrosinases and catechol oxidases. Six of the seven cysteines are involved in conserved disulfide bridges (Cys47-Cys393, Cys75-Cys134, and Cys196-Cys234) and expected to

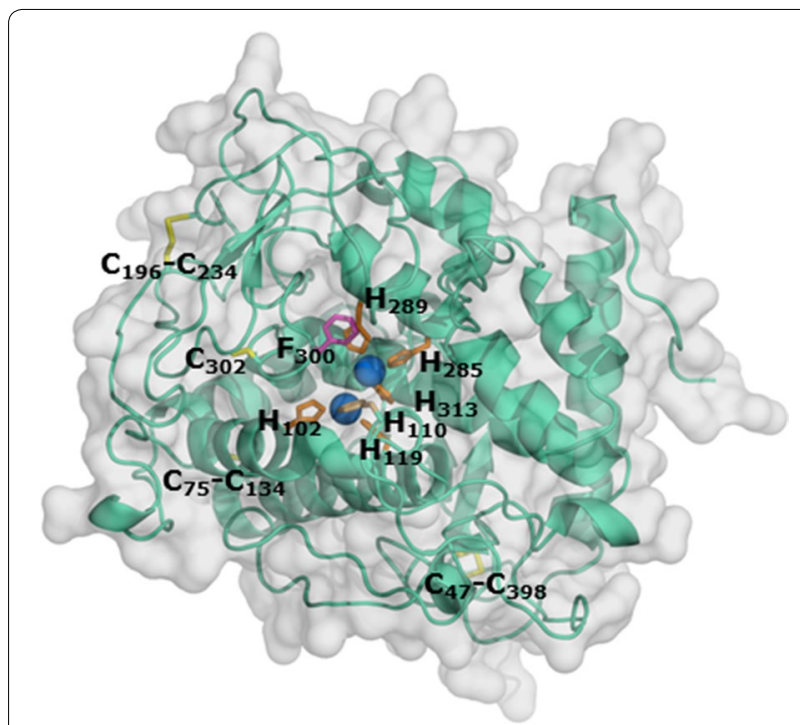

Fig. 5 Structural model of MtPPO7. The structural model of MtPPO7 was generated based on the crystal structure of a catechol oxidase from Aspergillus oryzae (AoCO4, Protein Data Bank entry: 4j3p) [42]. MtPPO7 and AoCO4 share an amino acid sequence identity of 38\%. The MtPPO7 model is predominantly a-helical with the catalytic copper site situated in the four-helix bundle. The coordination of the two copper atoms (b/ue) by six histidine residues (orange) is strictly conserved. The three disulfide bridges Cys47-Cys398, Cys75-Cys134, and Cys196-Cys234 (yellow) are also conserved be relevant for the thermo-tolerance of MtPPO7 (Fig. 5). Based on the model, the large distance (10.5 $\AA$ ) between the sulfur atom of Cys302 and $C \varepsilon$-atom of His110 prevents formation of a thioether bond, which is present in other PPOs such as AbPPO3 and AbPPO4 [25]. Characteristics of the amino acid sequence and structural model of $M t \mathrm{PPO} 7$ are further described in the "Discussion" section.

\section{Genome-wide analysis of AA9 LPMO, AbPPO, and MtPPO7}

Sequence annotations of 336 Ascomycota and 208 Basidiomycota genomes [29] were used in this analysis. Some of the numbers of genes obtained encoding AA9 LPMOs were verified by comparison with published data. For example, we have identified 22 and 18 genes encoding AA9 LPMOs in M. thermophila and T. terrestris, respectively, which is similar to previously reported results [30]. In total, 277 Ascomycota genomes and 178 Basidiomycota genomes contained AA9 LPMOs encoding genes (Fig. 6). The two PPOs used in this work are regarded as two different proteins due to their low sequence identity of $12 \%$. The two PPO classes used for the genome analysis were not further divided into short and long tyrosinases [31]. Over 90\% of the Ascomycota that comprise genes encoding AA9 LPMOs showed also the presence of genes encoding either $A b \mathrm{PPO}-$ like, $M t$ PPO7-like proteins or both (Fig. 6a). In contrast, only around $40 \%$ of the AA9 LPMOs encoding Basidiomycota contained genes that encode $A b \mathrm{PPO}-$ like, $M t \mathrm{PPO} 7$-like proteins or both. The percentage of Ascomycota and Basidiomycota genomes studied that contained neither genes encoding AA9 LPMOs, AbPPOs, nor $M t$ PPO7s were 15 and $8 \%$, respectively (Fig. 6a). The average number of genes found per genome encoding AA9 LPMOs was higher in Ascomycota (13.0) than in Basidiomycota (10.6) (Fig. 6b, c). Both the total and the average number of genes encoding AbPPO-like and MtPPO7-like proteins were also higher in Ascomycota compared to Basidiomycota (Fig. 6b, c).

Principal component analysis (PCA) was performed on all 336 Ascomycota and 208 Basidiomycota. In addition, we used the numbers which describe the presence of AA9 LPMO, MtPPO7s, and AbPPOs encoding genes in each fungal species.

The first two components of the PCA explained 69.8 and $19.7 \%$ variation in the data, respectively. Correlations of 0.75 and 0.5 were observed between the presence of genes encoding AA9 LPMOs and MtPPO7s in Ascomycota and Basidiomycota, respectively (Fig. 7b). Lower correlations of 0.59 and 0.34 were observed in the genes encoding AA9 LPMOs and AbPPOs. No correlation (correlation $\leq 0.25$ ) was observed between $A b P P O s$ and $M t \mathrm{PPO}$, for both Ascomycota and Basidiomycota. 

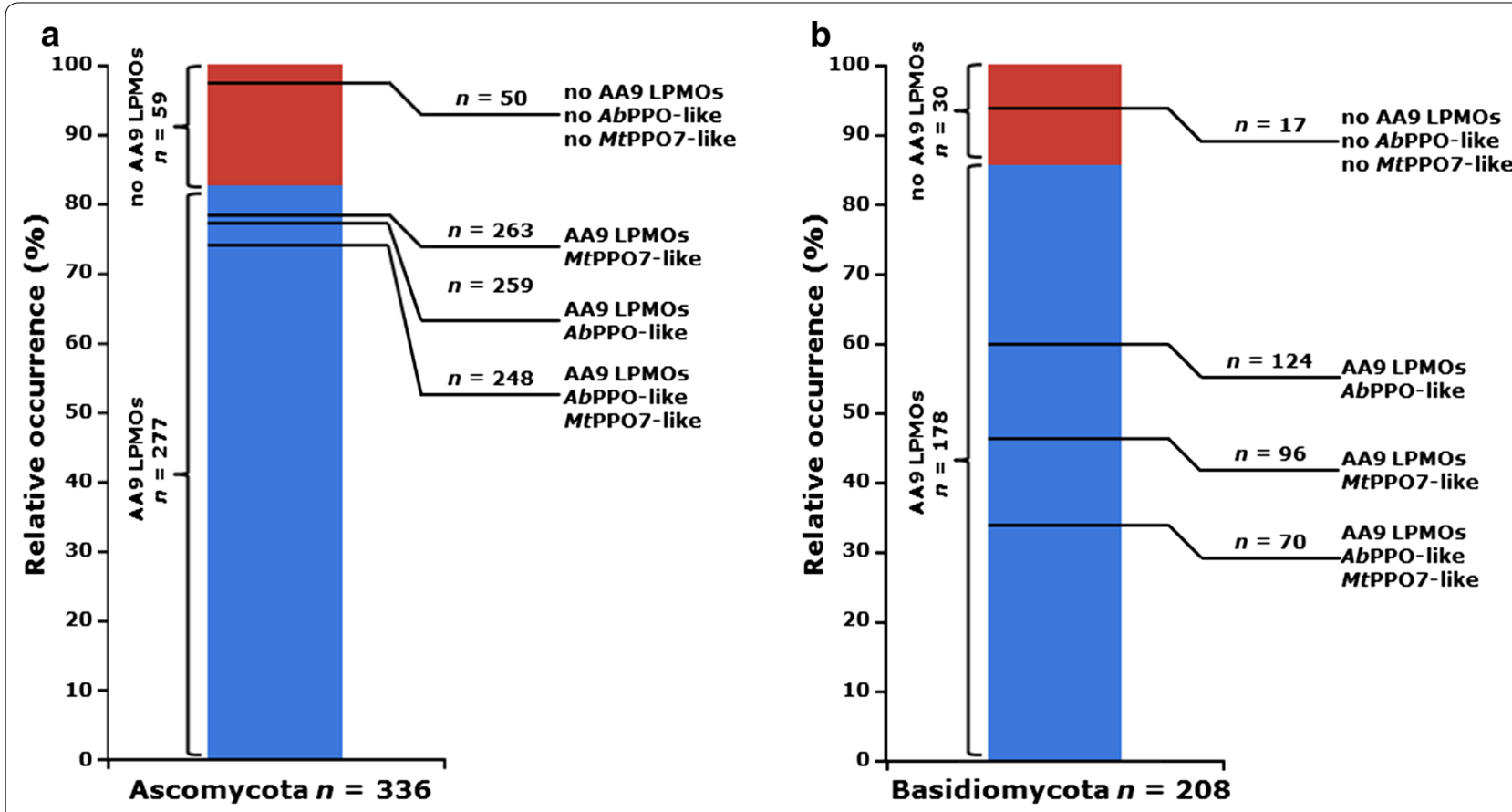

\begin{tabular}{c|c|c|c} 
& Ascomycota & Basidiomycota & Total \\
\hline AA9 LPMOs & 4,379 & 2,204 & 6,583 \\
AbPPO-like & 869 & 286 & 1,155 \\
MtPPO7-like & 2,020 & 494 & 2,514
\end{tabular}

\begin{tabular}{c|cc|cc|cc} 
& \multicolumn{2}{|c|}{ AA9 LPMOs } & \multicolumn{2}{c|}{ AbPPO-like } & \multicolumn{2}{c}{ MtPPO7-like } \\
& Ascomycota & Basidiomycota & Ascomycota & Basidiomycota & Ascomycota & Basidiomycota \\
\hline Minimum & 0 & 0 & 0 & 0 & 0 & 0 \\
Median & 8 & 8 & 2 & 1 & 5 & 1 \\
Mean & 13.0 & 10.6 & 2.6 & 1.4 & 6.0 & 2.4 \\
Maximum & 50 & 44 & 22 & 14 & 39 & 41
\end{tabular}

Fig. 6 Genome analysis of Ascomycota and Basidiomycota. The protein sequence annotations of 336 Ascomycota and 208 Basidiomycota genomes were used for genome analysis. In total, a 277 Ascomycota and b 178 Basidiomycota comprise AA9 LPMOs encoding genes (blue column). Next to this occurrence of AA9 LPMOs encoding genes in Ascomycota and Basidiomycota, the co-occurrence of both AbPPO-like and MtPPO7like, or either AbPPO-like or MtPPO7-like enzymes is indicated on the right of each column. The number of Ascomycota and Basidiomycota without co-occurrence of AA9 LPMOs encoding genes was 59 and 30, respectively (red column). In total, 50 Ascomycota and 17 Basidiomycota species were determined that do not carry any genes encoding AA9 LPMOs, AbPPOs, and MtPPO7s. c Total number of AA9 LPMOs, AbPPOs, and MtPPO7s encoding genes annotated in 336 Ascomycota and 208 Basidiomycota genomes. d Distribution of annotated AA9 LPMOs, AbPPOs, and MtPPO7s encoding genes within 336 Ascomycota and 208 Basidiomycota genomes. See "Methods" for details

Based on the presence of at least 10 annotated genes that encode cellulose-degrading enzymes per fungus, 27 Ascomycota and 23 Basidiomycota genomes were selected (Additional file 7: Table S2, Additional file 8: Table S3) [32]. The selected 27 Ascomycota genomes showed a higher correlation (0.60) between genes encoding AA9 LPMOs and MtPPO7s compared to the 23 selected Basidiomycota (0.53) (Fig. 7c). Also, no correlation was found between genes encoding MtPPO7- and $A b$ PPO-like genes in the selected Basidiomycota species (Fig. 7).

\section{Discussion}

MtPPO7 enhances cellulose oxidation by MtLPMO9B

Many PPOs, tyrosinases in particular, have been described to oxidize monophenols and various phenolic compounds comprising a 1,2-benzenediol or 1,2,3-benzenetriol moiety. However, none of these tyrosinases show a high activity towards methoxylated compounds (group Ib) such as ferulic acid (no. 8) [33-36]. Importantly, MtPPO7 hydroxylates methoxylated phenolic compounds (group $\mathrm{Ib}$ ) and thereby improves the activity of $M t$ LPMO9B up to 75 times (no. 5, Table 1). On 

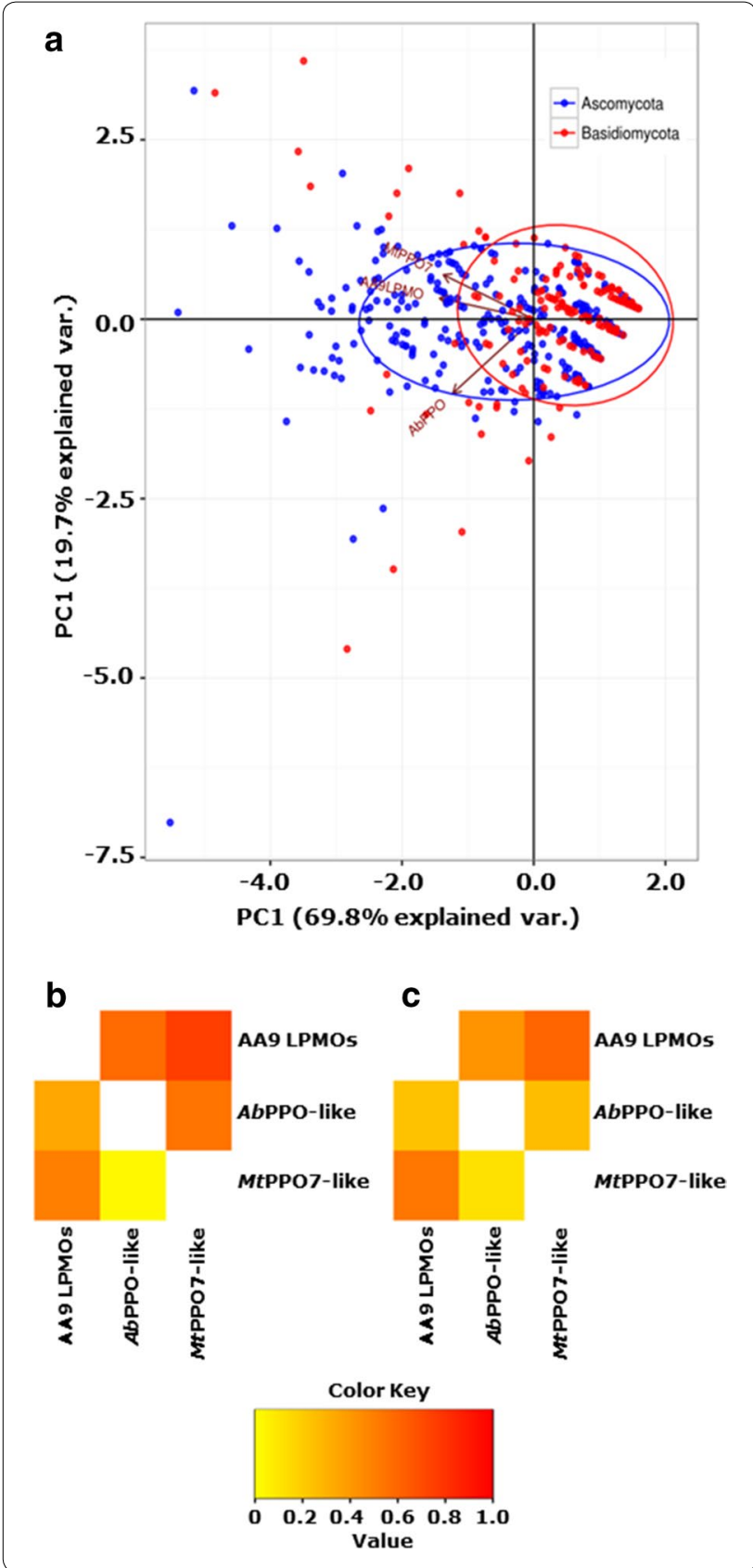

the other hand, $A b \mathrm{PPO}$ addition to RAC incubated with $M t$ LPMO9B in the presence non-methoxylated monophenols also resulted in a significant increase of the $M t$ LPMO9B activity (up to 99 times). Especially the activity of $M t \mathrm{PPO} 7$ towards methoxylated phenolic compounds is of high relevance, as these compounds are abundant as structural lignin units and, therefore, intrinsically present during plant biomass degradation. Here, we only determined the effect of polyphenol oxidases on the LPMO-mediated cellulose degradation in the
Fig. 7 Statistical genome analysis of Ascomycota and Basidiomycota. a Principal component analysis (PCA) of genes encoding AA9 LPMOs, AbPPOs, and MtPPO7s of 336 Ascomycota and 208 Basidiomycota genomes. The numbers which describe the presence of genes encoding AA9 LPMOs, MtPPO7s, and AbPPOs in each fungal species were used as variables and are presented as principal components of Ascomycota (red dots) and Basidiomycota (blue dots). The first two components of the PCA explained 69.8 and $19.6 \%$ variation in the data, respectively. Vectors (red arrows) with a similar orientation illustrate a high correlation between the gene families $(\mathbf{b})$. The axis legends indicate the overall contribution of the three gene families encoding AA9 LPMOs, AbPPOs, and MtPPO7s. b Correlation between the three gene families encoding AA9 LPMOs, AbPPOs, and MtPPO7s of 336 Ascomycota (upper triangle) and 208 Basidiomycota (lower triangle). c Correlation between genes encoding AA9 LPMOs, AbPPOs, and MtPPO7s of 27 Ascomycota (upper triangle) and 23 Basidiomycota (lower triangle), which have at least ten annotated genes encoding cellulose-degrading enzymes per fungus. Species of both fungal classes are listed in Additional file 7: Table S2 and Additional file 8: Table S3. Numerical values of the correlations of $\mathbf{b}$ and $\mathbf{c}$ are presented in Additional file 9: Figure S6. See "Methods" for details

presence of phenolic compounds that are known as lignin building blocks but we did not, for example, investigate the impact of these enzymes in the presence of washed or unwashed pretreated biomass. In comparison to the substrate used in this thesis, we expect a lower effect of $M t$ PPO7-like enzymes on the LPMO-mediated substrate oxidation using pretreated biomass, since this biomass contains a mixture of multiple potential electron-donating compounds, which were described in an earlier study [13].

A recent study described that $\mathrm{H}_{2} \mathrm{O}_{2}$, in addition to or instead of $\mathrm{O}_{2}$ (Fig. 1), acts as a co-substrate for LPMOs, while reducing agents are still needed to activate the active site copper [37]. Once copper is activated, LPMOs oxidize substrates under the use of $\mathrm{H}_{2} \mathrm{O}_{2}$. The latter can be formed by the reduction of $\mathrm{O}_{2}$ through reducing agents [37]. Considering these recent findings, it can only be hypothesized how MtPPO7 increased the MtLPMO9B-mediated cellulose oxidation, which was investigated in our research. It is possible that the oxidation of the phenolic compounds led to the formation of compounds that have either an enhanced reducing efficiency on $M t$ LPMO9B or led to an enhanced $\mathrm{H}_{2} \mathrm{O}_{2}$ generation which increased the $M t$ LPMO9B activity.

\section{Activity of MtPPO7 towards phenolic compounds}

Based on our results, we conclude that $M t \mathrm{PPO} 7$ improves the activity of $M t$ LPMO9B by two main reactions. First, MtPPO7 hydroxylates methoxylated monophenols at the ortho-position (monophenolase activity) and forms compounds comprising a 1,2-benzenediol moiety. These compounds have, compared to methoxylated monophenols, a lower redox potential and are known to be good 
electron donors for LPMOs [12-14]. Secondly, MtPPO7 inhibits the formation of non-enzymatic coupling reactions, which occurred during the incubation of several cinnamic acid derivatives in the absence of $M t \mathrm{PPO}$, but were not formed when $M t \mathrm{PPO} 7$ was present during the incubations (Additional file 4: Table S1). Non-enzymatic coupling reactions of cinnamic acid derivatives of group I, II, or III result in the formation of bulky phenolic polymers. The aliphatic acrylic acid (prop-2-enoic acid) group present in cinnamic acid derivatives constitutes an elongation of the conjugated aromatic ring. This group takes part in polymerization reactions, which can be caused by radical formation due to the presence of copper $(2.5 \mu \mathrm{M})$ $[18,38]$. However, it remains unclear, how MtPPO7 prevents (reduces) the polymerization of cinnamic acid derivatives.

Importantly, MtPPO7 showed only a low efficiency towards non-methoxylated compounds comprising a 1,2-benzenediol or 1,2,3-benzenetriol moiety. In contrast to $A b \mathrm{PPO}, M t \mathrm{PPO} 7$ had no inhibitory effect on the $M t$ LPMO9B activity towards $\mathrm{RAC}$ in the presence of these phenolic compounds (Table 1). Although we did not determine the formation of ortho-quinones, it is possible that $M t \mathrm{PPO}$ further oxidizes methoxylated compounds comprising a 1,2-benzenediol moiety into ortho-quinones due to the observed pigment formation (Additional file 5: Figure S4, Additional file 6: Figure S5). However, this pigment formation could also partly result from non-enzymatic polymerization reactions or formation of metal-catechol complexes, as indicated in Fig. 8 [39]. Still, based on the monophenolase activity we would consider $M t \mathrm{PPO} 7$ to be a tyrosinase. The low efficiency of $M t \mathrm{PPO} 7$ to convert compounds comprising a 1,2-benzenediol moiety is expected to be an advantage. $M t$ LPMO9B can utilize these compounds as electron donors and improve the oxidative activity towards cellulose. In the case of a strong diphenolase activity, however, the available amount of these compounds comprising a 1,2-benzenediol moiety would rapidly decrease due to further oxidation of these compounds into ortho-quinones. Hence, we expect for some other PPOs, such as $A b \mathrm{PPO}$, a negative effect on the LPMO-mediated cellulose oxidation. This effect results from the strong activity of these PPOs towards compounds comprising a 1,2-benzenediol or 1,2,3-benzenetriol moiety, which are potential reducing agents for LPMOs [33, 40, 41]. The ortho-quinones formed (no. 17 and 18) (Fig. 2; Table 1) have been shown to be less efficient electron donors for LPMOs than their ortho-diphenol precursors [12].

\section{Structural characteristics of $M t P P O 7$}

The structural model of $M t \mathrm{PPO} 7$ shows a four-helix bundle architecture with the presence of six histidine residues coordinating the two copper ions in the active site, which is typical for PPO-like tyrosinases and catechol oxidases (Fig. 5). We clearly showed that the preference

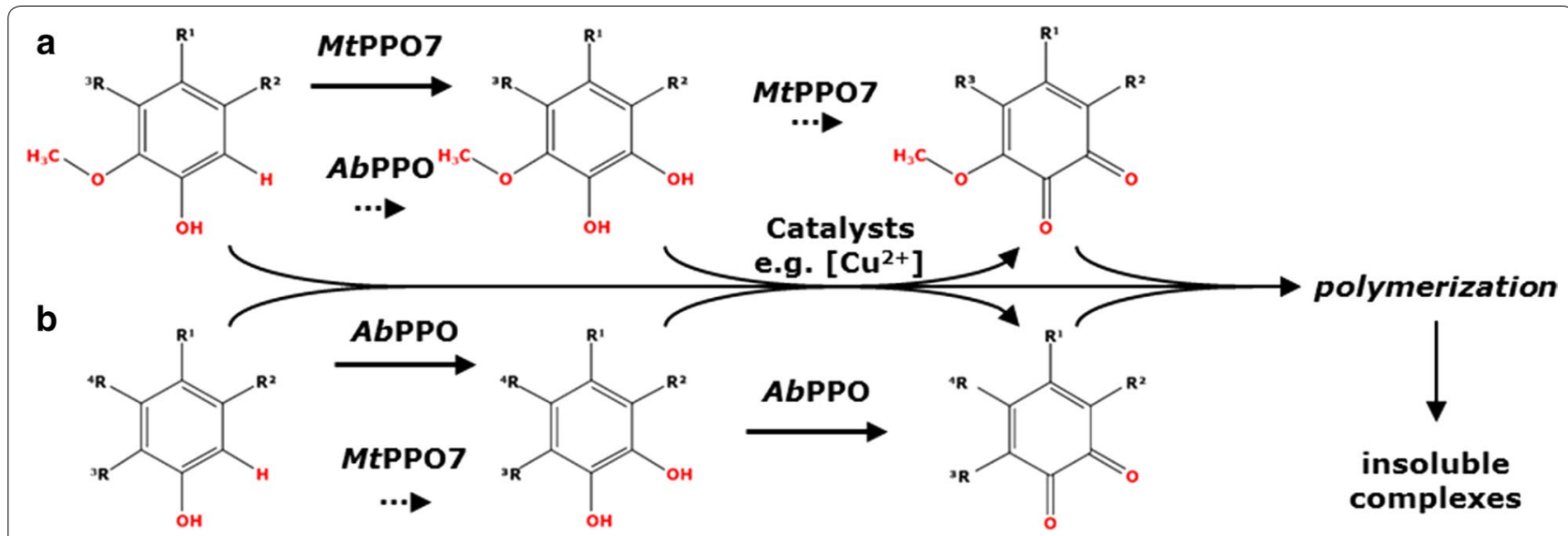

Fig. 8 Proposed reaction pathway of MtPPO7- and AbPPO-mediated oxidation of phenolic compounds. a Phenolic compounds comprising a 2-methoxy moiety are hydroxylated by MtPPO7 from M. thermophila C1 at the 6-position. Different from MtPPO7, AbPPO from A. bisporus shows a low efficiency (dotted arrows) towards these methoxylated monophenols. The formed compounds comprising a 1,2-dihydroxy-3-methoxy moiety have, compared to the methoxylated monophenols, a lower electron potential and an increased electron-donating capacity for LPMOs. b Nonmethoxylated monophenols are hydroxylated at the ortho-position by AbPPO into compounds comprising a 1,2-benzenediol moiety, whereas MtPPO7 shows a low efficiency (dotted arrows) towards non-methoxylated monophenols. The formed compounds comprising a a 1,2-dihydroxy3-methoxy and $\mathbf{b}$ 1,2-benzenediol moiety are expected to be further oxidized into ortho-quinones by either the MtPPO7-and AbPPO-mediated or catalyst-mediated oxidation, such as copper which was present during the incubation. As indicated in Additional file 6: Figure S5, these orthoquinones, including intermediate products formed by the oxidation of phenolic compounds, are expected to polymerize and form insoluble complexes 
of $M t \mathrm{PPO} 7$ towards methoxylated phenolic compounds and the low activity towards diphenols is different from reported fungal or plant catechol oxidases and tyrosinases (Fig. 4). Here, we further discuss the structurefunction relationship of these PPOs in comparison with the generated model of MtPPO7. However, it should be noted that the structural model of MtPPO7 was generated based on a catechol oxidase from Aspergillus ory$z a e(A o C O 4)$ and both proteins share an overall amino acid sequence identity of only $38 \%$ [42]. It has already been shown that marginal structural differences determine whether PPOs exhibit a stronger monophenolase or diphenolase activity [43]. Based on our model, it is possible that structural features discussed below deviate compared to the crystal structure which is, however, not available yet.

Firstly, the presence of a thioether bridge between a cysteine and histidine is assumed to be important for the monophenolase and diphenolase activity. This bond has been described to be present in many fungal PPOs, such as $A b \mathrm{PPO} / 4$ from $A$. bisporus, $N c \mathrm{PPO}$ from Neurospora crassa, and also in plant catechol oxidases, such as $I b C O$ from Ipomoea batatas [26, 44, 45]. However, similar to AoCO4 from A. oryzae [42], MtPPO7 does not form this thioether bond (Fig. 5). Secondly, MtPPO7 shows the presence of the PPO typical 'gate residue' (Phe300 in MtPPO7, Phe261 in IbCO, and Val299 in AoCO4). It has been hypothesized that a bulky 'gate residue', such as a phenylalanine, limits the accessibility of monophenols to the copper $\mathrm{A}$ ion $(\mathrm{CuA})$ and, therefore, might be a limiting factor for the monophenolase activity $[44,46]$. However, in $\mathrm{AoCO} 4$ the bulky phenylalanine is replaced by Val299 and still AoCO4 shows a rather low activity towards monophenols [31, 42]. Our data suggest that Phe300 in MtPPO7 does not limit the accessibility for (methoxylated) monophenols. Thirdly, it also has recently been proposed that the presence of certain amino acid residues next to the $\mathrm{CuB}$-coordinating histidines indicates whether a tyrosinase or catechol oxidase exhibits a stronger monophenolase and a weak diphenolase activity or a weak monophenolase and a strong diphenolase activity [43]. However, none of the described amino acid residues at that specific position (Pro, Asn, Glu, and Gln) is present in MtPPO7, which has a tyrosine (Tyr268) at the corresponding position. In $M t \mathrm{PPO} 7$ a tyrosine (Tyr268) occupies. Whether Tyr268 is responsible for the observed specificity towards methoxylated monophenols can only be hypothesized at this moment. Fourthly, MtPPO7 shares with tyrosinases and catechol oxidases the characteristic 'tyrosine motif' (Tyr-X-Tyr/Phe or Tyr/ Phe-X-Tyr; in MtPPO7 residues 399-401). This motif is highly conserved among plants and fungi [31, 42, 47]. Fifthly, MtPPO7 does not contain the 'YG motif' (Gly-Tyr motif), which is a typical feature of fungal tyrosinases and catechol oxidases [47]. In conclusion, based on substrate specificity (Fig. 1) and modeled structure (Fig. 5), we do not classify $M t \mathrm{PPO} 7$ as a typical tyrosinase or catechol oxidase.

\section{Genome analysis}

Based on genome analysis, we found a positive correlation between genes encoding AA9 LPMOs and MtPPO7like enzymes in Ascomycota and Basidiomycota, which has not been shown previously. Interestingly, this correlation strengthens the evidence that fungi benefit from the concerted activity of AA9 LPMOs and MtPPO7-like enzymes in nature, which is in agreement with major findings obtained from the experimental data of this work. The proportion of annotated AA9 LPMOs within Ascomycota and Basidiomycota (82 and $86 \%$, respectively) is about $5-10 \%$ lower with the findings of a recent study (92\% in Ascomycota and Basidiomycota), which may result from the different species chosen (Fig. 6) [12]. The two PPOs (MtPPO7 and AbPPO) used in this work share only a low sequence identity and, therefore, are considered to belong to different subgroups of polyphenol oxidases. It is important to realize that active tyrosinases, such as the mushroom tyrosinase $A b \mathrm{PPO} 3$, have been described to consist of large $(\mathrm{H})$ and small $(\mathrm{L})$ subunits $[45,48]$. We focused for the genome analysis on genes encoding the protein of the catalytic $\mathrm{H}$ subunit, which contains the highly conserved binuclear copper site. The function of the $L$ subunit is so far not known [45]. All purified enzymes used in this work are secreted. However, we did not differentiate between secreted and non-secreted fungal AA9 LPMOs and PPOs in the genome analysis. It may be that most of the enzymes detected here take part in the external fungal metabolism, as can be deducted from the high abundance of the annotated $M t$ PPO7s and AbPPOs throughout Ascomycota and Basidiomycota. We, therefore, specifically selected genomes of plant biomass degrading fungi that contain at least ten genes encoding known cellulose-degrading enzymes per fungus and found again a similar correlation between genes encoding AA9 LPMOs, AbPPOs, and $M t$ PPO7s (Fig. 7) [32, 49]. In contrast to $M t P P O 7 s$, genes encoding $A b$ PPOs showed a weak correlation with AA9 LPMOs in Ascomycota and Basidiomycota (Fig. 7), especially for the selected cellulose-degrading Basidiomycota (Fig. 7c). One possible explanation is that a cellulose-rich environment is associated with the abundance of lignin as seen in soft- and hardwoods. Still, there may be considerable variation in the substrate specificity and specific activity of PPOs within the MtPPO7- or AbPPO-like gene families. The latter seems to be consistent with the low sequence identity among PPOs and, in addition, by 
the diverse substrate specificities of PPOs that have only marginal differences in their amino acid sequence [31, 43, 50].

\section{Conclusions}

For the first time, we demonstrate the importance of the coupled action of different monooxygenases in the concerted degradation of plant biomass. We demonstrated that $M t \mathrm{PPO} 7$ is particularly active towards methoxylated phenolic compounds that are the predominant structural units of lignin. This feature distinguishes $M t \mathrm{PPO} 7$ from the well-known mushroom tyrosinase $A b \mathrm{PPO}$, which stimulates the LPMO activity via its ability to hydroxylate non-methoxylated monophenols. However, its strong diphenolase activity limits the applicability of this tyrosinase for producing electron-donating capacity for LPMOs. In addition, we established that genes encoding MtPPO7-like enzymes and AA9 LPMOs are highly correlated throughout Ascomycota and Basidiomycota, suggesting that AA9 LPMOs benefit from the activity of $M t$ PPO7-like enzymes in nature. Further understanding in both lignin deconstruction and enzymatic lignocellulose oxidation will lead to more eco-friendly routes for biomass utilization, which is seen as a prerequisite for a circular economy.

\section{Methods}

\section{Enzyme expression, production, and purification}

MtPPO7 (UniProt: KX772412) was over-expressed in the homologous Myceliophthora thermophila $\mathrm{C} 1$ strain. A low protease/low (hemi-)cellulase-producing $M$. thermophila $\mathrm{C} 1$ strain was used to produce MtPPO7 [51, 52]. The MtPPO7-containing culture broth was fractionated to obtain a pure $M t \mathrm{PPO} 7$ preparation. This preparation was provided by DuPont Industrial Biosciences. MtLPMO9B was expressed and purified as described in [13].

\section{Protein identification}

The pure $M t \mathrm{PPO} 7$ fraction was analyzed by LC-mass spectrometry confirming the presence of the $M t \mathrm{PPO} 7$ by 'The Scripps Research Institute' (San Diego, CA, USA).

\section{Purification and identification of mushroom tyrosinase}

Tyrosinase from the edible button mushroom Agaricus bisporus was purified from a commercial enzyme preparation (Sigma-Aldrich, Steinheim, Germany) as described previously [25]. The purified enzyme preparation (referred to as $A b \mathrm{PPO}$ ) was shown to contain the isoforms PPO3 and PPO4 [25].

\section{Cellulose substrate and reducing agents}

Regenerated amorphous cellulose (RAC) was prepared from Avicel PH-101 as described previously [7, 53]. All reducing agents used throughout this study (Table 1) were purchased from Sigma-Aldrich (Steinheim, Germany).

Incubation conditions for MtLPMO9B, MtPPO7, and AbPPO Regenerated amorphous cellulose $\left(1-2 \mathrm{mg} \mathrm{mL}^{-1}\right.$, see Figure captions) was dissolved in $50 \mathrm{mM}$ ammonium acetate buffer $(\mathrm{pH}=5.0)$, with or without addition of reducing agents (final concentration of $2 \mathrm{mM}$ ). The standard enzyme concentrations of $M t \mathrm{LPMO} 9 \mathrm{~B}, M t \mathrm{PPO}$, and $A b$ PPO used in this work were 5.0, 5.0, and $0.7 \mu \mathrm{g}$ protein $\mathrm{mg}^{-1}$ substrate, respectively. All samples were incubated for $20 \mathrm{~h}$ at $50^{\circ} \mathrm{C}$ in a head-over-tail rotator in portions of $1 \mathrm{~mL}$ total volume (Stuart rotator, Bibby Scientific, Stone, UK) at $20 \mathrm{rpm}$. Supernatants of all incubations were analyzed by HPAEC and RP-UHPLC-UV-ESI-MS ${ }^{\mathrm{n}}$.

\section{Oligosaccharide analysis}

Oligosaccharides were analyzed by high-performance anion-exchange chromatography (HPAEC) with pulsed amperometric detection (PAD) using a HPAEC system (ICS-5000, Dionex, Sunnyvale, CA, USA) as described previously [7].

\section{RP-UHPLC-UV-ESI-MS ${ }^{\mathrm{n}}$ analysis}

Supernatants of all incubations were subjected to an Accela reversed-phase high-performance liquid chromatography (RP-UHPLC) system coupled to electron spray ionization mass spectrometry (Thermo Scientific, San Jose, CA, USA). Injected samples $(5 \mu \mathrm{L})$ were separated using an Acquity C18 column $(2.1 \times 150 \mathrm{~mm}, 1.7 \mu \mathrm{m}$ particle size) with an Acquity UHPLC Shield RP18 Vanguard guard column $(2.1 \times 5 \mathrm{~mm}, 1.7 \mu \mathrm{m}$ particle size $)$. Both columns were purchased from Waters (Milford, MA, USA). Gradient elution with eluent $\mathrm{A}\left(\mathrm{H}_{2} \mathrm{O}+1 \%\right.$ $(\mathrm{v} / \mathrm{v})$ acetonitrile $+0.1 \%(\mathrm{v} / \mathrm{v})$ HOAc) and eluent $\mathrm{B}$ (acetonitrile $+0.1 \%(\mathrm{v} / \mathrm{v}) \mathrm{HOAc})$ was performed according to the following steps: From 0 to 17.7 min a linear gradient from 5 to $60 \% \mathrm{~B}$; from 17.7 to $21.7 \mathrm{~min}$, isocratic $100 \%$ $\mathrm{B}$, and from 21.7 to $26 \mathrm{~min}$, isocratic $5 \% \mathrm{~B}$. The flow rate and the injection volume were $0.4 \mathrm{~mL} \mathrm{~min}^{-1}$ and $5 \mu \mathrm{L}$, respectively. The column temperature was set to $30{ }^{\circ} \mathrm{C}$ and the photodiode array detector was operated in the range of $200-400 \mathrm{~nm}$.

Samples were further analyzed using an LTQ-Velos mass spectrometer (Thermo Scientific) equipped with a ESI-MS. Data were collected over a $\mathrm{m} / \mathrm{z}$ range of 90-1500 in both negative (NI) and positive (PI) modes. The collision energy was set to $35 \%$.

\section{Structural modeling}

An alignment was made of the amino acid sequence of $M t \mathrm{PPO} 7$ and the amino acid sequence of catechol oxidase 
from Aspergillus oryzae (AoCO4), which scored highest in a Blast search using the $M t \mathrm{PPO}$ sequence against the Protein Data Bank (38\% amino acid identity). Using this alignment and the available structure of AoCO4 (PDB id: 4J3P) as template, structural models were obtained for $M t \mathrm{PPO} 7$ using the Modeller program version 9.16. One hundred comparative models were generated, after which the model with lowest corresponding DOPE score was selected for inspection and image generation using Pymol (Pymol, The PyMOL Molecular Graphics System, version 1.5.0.4 Schrödinger, LLC, New York, NY, USA).

\section{Genome-wide analysis}

Fungal genomes were obtained from the JGI MycoCosm portal [29]. In total, protein sequence annotations of 336 Ascomycota and 208 Basidiomycota genomes were used. BLAST databases for those protein sequences were created using 'makeblastdb' program in BLAST + v2.2.30 [54]. The protein BLAST was performed separately using ten $M t$ PPO7 sequences [PDB id: 4j3p and closely related MtPPO7s: Q2UNF9, Aspergillus oryzae (strain ATCC 42149); G2QC95, Myceliophthora thermophila (ATCC 42464); Q2H7I7, Chaetomium globosum (ATCC 6205); GOSFX8, Chaetomium thermophilum (DSM 1495); L7IAQ4, Magnaporthe oryzae (strain Y34); L7JMT9, Magnaporthe oryzae (strain P131); G4N2I5, Magnaporthe oryzae (strain 70-15); A0A084GCK1, Scedosporium apiospermum; A0A0C4DYF2, Magnaporthiopsis poae (ATCC 64411); J3P591, Gaeumannomyces graminis var. tritici (strain R3-111a-1)], ten AA9 LPMO sequences (PDB id: $4 \mathrm{~d} 7 \mathrm{u}$ and closely related LPMOs: Q7SHI8, Neurospora crassa (strain ATCC 24698); G2QCJ3, Myceliophthora thermophila (strain ATCC 42464); F7W1P4, Sordaria macrospora (strain ATCC MYA-333); G2RB73, Thielavia terrestris (strain ATCC 38088); Q2H8N9, Chaetomium globosum (strain ATCC 6205); G0S408, Chaetomium thermophilum (strain DSM 1495); F8MLY8, Neurospora tetrasperma (strain FGSC 2508); T0L448, Colletotrichum gloeosporioides (strain Cg-14); A0A0H4K9X4 and A0A1C9CXI0, Myceliophthora thermophila $\mathrm{C} 1$ ); and four AbPPOs (AbPPO1, AbPPO2, AbPPO3, and AbPPO4 $[55,56])$ as query sequences. Resulting sequences below E-value cut-off of 0.001 with query coverage above $60 \%$ for AA9 LPMOs, $65 \%$ for $M t$ PPO7s, and $85 \%$ for $A b P P O s$ were considered for further analysis. Selection of cellulase-rich Ascomycota and Basidiomycota was based on the presence of at least 10 genes encoding cellulose-degrading enzymes, which are classified in the CAZy database as glycosyl hydrolase families GH1, GH3, GH5, GH6, GH7, GH12, GH45. The GH gene families were selected based on Kubicek et al. [57]. Previous data [32] were used to determine the number of annotated genes encoding cellulose-degrading enzymes. Based on this selection, 27 Ascomycota and 23 Basidiomycota species were selected for the Pearson correlation analysis (Fig. 7; Additional file 7: Table S2, Additional file 8: Table S3). All the statistical analyses were performed in $\mathrm{R}$ [58].

\section{Additional files}

Additional file 1: Figure S1. Activity of MtLPMO9B towards amorphous cellulose in the presence and absence of MtPPO7 or AbPPO. HPAEC elution pattern of regenerated amorphous cellulose (RAC; $1.5 \mathrm{mg} \mathrm{mL}^{-1}$ ) incubated with MtLPMO9B (red, $5.0 \mathrm{\mu g} \mathrm{mL}^{-1}$ ) only, or with either AbPPO (blue, $2.5 \mu \mathrm{L} \mathrm{mL}^{-1}$ ) or MtPPO7 (yellow, $5.0 \mu \mathrm{g} \mathrm{mL}^{-1}$ ) in the presence of (a) para-coumaric acid (no. 3 specified in Table 1, 2 mM) and (b) 3-hydroxy4-methoxycinnamic acid (no. 5 specified in Table 1, 2 mM). The incubation of RAC with MtLPMO9B results in the formation of non-oxidized gluco-oligosaccharides $\left(\mathrm{GlCOS}_{n}\right.$ ) and C1-oxidized gluco-oligosaccharides $\left(\mathrm{GlCOS}_{n}^{\#}\right)$. See "Methods" for details.

Additional file 2: Figure S2. Release of oligosaccharides from RAC incubated with MtLPMO9B in the presence and absence of MtPPO7 throughout $24 \mathrm{~h}$. Samples were incubated in the presence of ferulic acid (no. 8 specified in Table 1). The total sum is shown as integrated peak areas of released non-oxidized (shaded red and shaded yellow) and (1-oxidized (red and yellow) gluco-oligosaccharides after incubation of regenerated amorphous cellulose (RAC; $1.5 \mathrm{mg} \mathrm{mL}^{-1}$ ) with MtLPMO9B only (red bars, $5 \mathrm{mg} \mathrm{mL}^{-1}$ ) and MtLPMO9B together with MtPPO7 (yellow bars, $5 \mathrm{mg}$ $\mathrm{mL}^{-1}$ ) based on HPAEC. All incubations were performed in duplicate, and the standard deviations are presented as error bars. See "Methods" for details.

Additional file 3: Figure S3. Concentration of phenolic compounds incubated in the presence and absence of MtPPO7. (a) guaiacol (no. 9 specified in Table 1, $2 \mathrm{mM}$ ) and (c) 3-methylcatechol (no. 17 specified in Table 1,2 mM) were incubated with MtPPO7 (yellow bar, $5 \mathrm{\mu g} \mathrm{mL}^{-1}$ ) or without (red bar). Samples were incubated in a $50 \mathrm{mM}$ potassium phosphate buffer ( $\mathrm{pH}=6.0$ ) containing $2.5 \mu \mathrm{M}$ copper(II)-chloride for $24 \mathrm{~h}$ at $50{ }^{\circ} \mathrm{C}$. The conversion of guaiacol and 3-methylcatechol by MtPPO7 was calculated by subtracting the determined concentration of the incubation of guaiacol or 3-methylcatechol in the presence of MtPPO7 from the concentration that was determined by the incubation of guaiacol and 3-methylcatechol alone. This conversion was expressed as the relative decrease of the guaiacol and 3-methylcatechol concentration and is shown in (b) and (d), respectively. See "Methods" for details.

Additional file 4: Table S1. Compounds detected after incubation of 21 reducing agents without or with MtPPO7 by UHPLC/UV-MS ${ }^{1}$

Additional file 5: Figure S4. UHPLC-UV-MS ${ }^{n}$ elution profile of guaiacol incubated (a) in the presence and (b) absence of MtPPO7. Guaiacol (no. 9 specified in Table 1,2 mM) was incubated with $\left(5 \mu \mathrm{g} \mathrm{mL}^{-1}\right)$ or without MtPPO7. Samples were incubated in a $50 \mathrm{mM}$ potassium phosphate buffer $(\mathrm{pH}=6.0)$ containing $2.5 \mu \mathrm{M}$ copper(II)-chloride for $24 \mathrm{~h}$ at $50^{\circ} \mathrm{C}$. Annotation of the peaks based on UV was done by using mass spectrometry (Additional file 6: Figure S5). See "Methods" for details.

Additional file 6: Figure S5. Schematic presentation of possible reaction pathways of guaiacol incubated in the presence of MtPPO7. In short, MtPPO7 hydroxylates guaiacol (no. 9 specified in Table 1) into 3-methoxycatechol (monophenolase activity). Although not determined, it is likely that 3-methoxycatechol is further oxidized by MtPPO7 into the corresponding ortho-quinone (diphenolase activity, dashed arrow). These ortho-quinones are expected to polymerize and form insoluble complexes. Guaiacol itself forms insoluble complexes via auto-oxidation, which results from the presence of copper during the incubation for $24 \mathrm{~h}$ at $50^{\circ} \mathrm{C}$. The decrease in guaiacol concentration during the incubation of guaiacol without MtPPO7 is also shown in Additional file 3: Figure S3a. The determined masses indicate the presence of multiple trimers (399.06, 401.06 and 415.04) consisting of polymerized 3-methylcatechol and 
guaiacol (Additional file 5: Figure S4). As described above, the polymerization reactions are expected to be catalyzed by copper during the incubation conditions applied. All masses were determined by UHPLC-UV-MS ${ }^{n}$ after incubation of guaiacol (2 mM) with MtPPO7 $\left(5.0 \mu \mathrm{g} \mathrm{mL}^{-1}\right)$. Samples were incubated for $24 \mathrm{~h}$ at $50^{\circ} \mathrm{C}$ in $50 \mathrm{mM}$ potassium phosphate, $\mathrm{pH} 6.0$ containing $2.5 \mu \mathrm{M}$ copper(II)-chloride.

Additional file 7: Table S2. Selected cellulase-rich Ascomycota from the JGI database?.

Additional file 8: Table S3. Selected cellulase-rich Basidiomycota from the JGI database 1 .

Additional file 9: Figure S6. Correlation of AA9 LPMOs, AbPPOs and MtPPO7s encoding genes in Ascomycota and Basidiomycota. Correlation between the three gene families encoding AA9 LPMOs, AbPPOs and MtPPO7s of (a) 336 Ascomycota and (b) 208 Basidiomycota. Correlation between genes encoding AA9 LPMOs, AbPPOs and MtPPO7s of (c) 27 Ascomycota and (d) 23 Basidiomycota, which have at least ten annotated genes encoding cellulose-degrading enzymes. Species of selected fungal classes are listed in Additional file 7: Table S2 and Additional file 8: Table S3. Graphical presentations of the correlations of (a) together with (b) and (c) and (d) are shown in Fig. 7. See "Methods" for details.

\section{Authors' contributions}

MF, MJK, JV, SWAH, DW, J-PV, and MAK designed research; MF performed research and analyzed data; SKM and MF performed genome analysis; AHW and MF performed structural modeling; and MF, SKM, AHW, WJHB, HG, and MAK wrote the paper. All authors read and approved the final manuscript.

\section{Author details}

${ }^{1}$ Laboratory of Food Chemistry, Wageningen University \& Research, Bornse Weilanden 9, 6708 WG Wageningen, The Netherlands. ${ }^{2}$ Laboratory of Biochemistry, Wageningen University \& Research, Stippeneng 4, 6708 WE Wageningen, The Netherlands. ${ }^{3}$ DuPont Industrial Biosciences, Nieuwe Kanaal 7-S, 6709 PA Wageningen, The Netherlands. ${ }^{4}$ Fungal Genetics \& Technology Consultancy, P.O. Box 39b, 6700 AJ Wageningen, The Netherlands.

\section{Acknowledgements}

This research was funded by The Graduate School VLAG (Advanced Studies in Food Technology, Agrobiotechnology, Nutrition and Health Sciences) of Wageningen University \& Research (Wageningen, The Netherlands) and by a VICI Grant (865.14.001) of the Netherlands Organization for Scientific Research (NWO) to D.W

\section{Competing interests}

The authors declare that they have no competing interests.

\section{Availability of supporting data}

Supporting data will be provided as Additional files 1, 2, 3, 4, 5, 6, 7, 8, 9 .

\section{Publisher's Note}

Springer Nature remains neutral with regard to jurisdictional claims in published maps and institutional affiliations.

Received: 26 January 2017 Accepted: 3 May 2017

Published online: 10 May 2017

\section{References}

1. Lam TBT, Kadoya K, liyama K. Bonding of hydroxycinnamic acids to lignin: ferulic and $p$-coumaric acids are predominantly linked at the benzyl position of lignin, not the $\beta$-position, in grass cell walls. Phytochemistry. 2001;57(6):987-92. doi:10.1016/50031-9422(01)00052-8.

2. Takahashi N, Koshijima T. Ester linkages between lignin and glucuronoxyIan in a lignin-carbohydrate complex from beech (Fagus crenata) wood. Wood Sci Technol. 1988;22(231-241):231-41. doi:10.1007/BF00386018.
3. Vaaje-Kolstad G, Westereng B, Horn SJ, Liu Z, Zhai H, Sorlie M, et al. An oxidative enzyme boosting the enzymatic conversion of recalcitrant polysaccharides. Science. 2010;330(6001):219-22. doi:10.1126/ science.1192231.

4. Lombard V, Golaconda Ramulu H, Drula E, Coutinho PM, Henrissat B. The carbohydrate-active enzymes database (CAZy) in 2013. Nucleic Acids Res. 2014:42;D490-5. doi:10.1093/nar/gkt1178.

5. Phillips CM, Beeson WT, Cate JH, Marletta MA. Cellobiose dehydrogenase and a copper-dependent polysaccharide monooxygenase potentiate cellulose degradation by Neurospora crassa. ACS Chem Biol. 2011;6(12):1399-406. doi:10.1021/cb200351y.

6. Quinlan RJ, Sweeney MD, Lo Leggio L, Otten H, Poulsen JC, Johansen $\mathrm{KS}$, et al. Insights into the oxidative degradation of cellulose by a copper metalloenzyme that exploits biomass components. Proc Natl Acad Sci USA. 2011;108(37):15079-84. doi:10.1073/pnas.1105776108.

7. Frommhagen M, Sforza S, Westphal AH, Visser J, Hinz SW, Koetsier MJ, et al. Discovery of the combined oxidative cleavage of plant xylan and cellulose by a new fungal polysaccharide monooxygenase. Biotechnol Biofuels. 2015;8:101. doi:10.1186/s13068-015-0284-1.

8. Vu WV, Beeson WT, Span EA, Farquhar ER, Marletta MA. A family of starch-active polysaccharide monooxygenases. Proc Natl Acad Sci USA. 2014;111(38):13822-7. doi:10.1073/pnas.1408090111.

9. Isaksen T, Westereng B, Aachmann FL, Agger JW, Kracher D, Kittl R, et al. A C4-oxidizing lytic polysaccharide monooxygenase cleaving both cellulose and cello-oligosaccharides. J Biol Chem. 2014;289(5):2632-42. doi:10.1074/jbc.M113.530196.

10. Forsberg Z, Rohr AK, Mekasha S, Andersson KK, Eijsink VG, Vaaje-Kolstad $\mathrm{G}$, et al. Comparative study of two chitin-active and two celluloseactive AA10-type lytic polysaccharide monooxygenases. Biochemistry. 2014;53(10):1647-56. doi:10.1021/bi5000433.

11. Agger JW, Isaksen T, Varnai A, Vidal-Melgosa S, Willats WG, Ludwig R, et al. Discovery of LPMO activity on hemicelluloses shows the importance of oxidative processes in plant cell wall degradation. Proc Natl Acad Sci USA. 2014;111(17):6287-92. doi:10.1073/pnas.1323629111.

12. Kracher D, Scheiblbrandner S, Felice AKG, Breslmayr E, Preims M, Ludwicka K, et al. Extracellular electron transfer systems fuel cellulose oxidative degradation. Science. 2016. doi:10.1126/science.aaf3165.

13. Frommhagen M, Koetsier MJ, Westphal AH, Visser J, Hinz SWA, Vincken $J-P$, et al. Lytic polysaccharide monooxygenases from Myceliophthora thermophila $\mathrm{C} 1$ differ in substrate preference and reducing agent specificity. Biotechnol Biofuels. 2016;9(1):1-17. doi:10.1186/s13068-016-0594-y.

14. Westereng B, Cannella D, Wittrup Agger J, Jørgensen H, Larsen Andersen M, Eijsink VGH, et al. Enzymatic cellulose oxidation is linked to lignin by long-range electron transfer. Scientific Rep. 2015;5:18561. doi:10.1038/ srep18561.

15. Bissaro B, Forsberg Z, Ni Y, Hollmann F, Vaaje-Kolstad G, Eijsink VGH. Fueling biomass-degrading oxidative enzymes by light-driven water oxidation. Green Chem. 2016;18:5357-66. doi:10.1039/c6gc01666a.

16. Cannella D, Mollers KB, Frigaard NU, Jensen PE, Bjerrum MJ, Johansen $\mathrm{KS}$, et al. Light-driven oxidation of polysaccharides by photosynthetic pigments and a metalloenzyme. Nat Commun. 2016;7:11134. doi:10.1038/ ncomms 11134

17. Gao Z-J, Liu J-B, Xiao X-G. Purification and characterisation of polyphenol oxidase from leaves of Cleome gynandra L. Food Chem. 2011;129(3):10128. doi:10.1016/j.foodchem.2011.05.062.

18. Dec J, Haider K, Bollag J-M. Decarboxylation and demethylation of naturally occurring phenols during coupling reactions and polymerization. Soil Sci. 2001;166(10):660-71.

19. Kuijpers TFM, van Herk T, Vincken J-P, Janssen RH, Narh DL, van Berkel WJH, et al. Potato and mushroom polyphenol oxidase activities are differently modulated by natural plant extracts. J Agric Food Chem. 2014;62(1):214-21. doi:10.1021/jf4043375.

20. Beloqui A, Pita M, Polaina J, Martínez-Arias A, Golyshina OV, Zumárraga $\mathrm{M}$, et al. Novel polyphenol oxidase mined from a metagenome expression library of bovine rumen: biochemical properties, structural analysis, and phylogenetic relationships. J Biol Chem. 2006;281 (32):22933-42. doi:10.1074/jbc.M600577200.

21. Lerch K. Neurospora tyrosinase: structural, spectroscopic and catalytic properties. Mol Cell Biochem. 1983;52(2):125-38. doi:10.1007/ bf00224921. 
22. Konrad L. Tyrosinase: molecular and active-site structure. Enzymatic browning and its prevention. ACS Symposium Series: American Chemical Society; 1995. p. 64-80

23. Vanholme R, Demedts B, Morreel K, Ralph J, Boerjan W. Lignin biosynthesis and structure. Plant Physiol. 2010;153(3):895-905. doi:10.1104/ pp.110.155119.

24. Kuijpers TFM, Narváez-Cuenca C-E, Vincken J-P, Verloop AJW, van Berkel WJH, Gruppen H. Inhibition of enzymatic browning of chlorogenic acid by sulfur-containing compounds. J Agric Food Chem. 2012;60(13):350714. doi:10.1021/jf205290w.

25. Kuijpers TFM, Gruppen H, Sforza S, van Berkel WJH, Vincken J-P. The antibrowning agent sulfite inactivates Agaricus bisporus tyrosinase through covalent modification of the copper-B site. FEBS J. 2013;280(23):6184-95. doi:10.1111/febs.12539.

26. Mauracher SG, Molitor C, Al-Oweini R, Kortz U, Rompel A. Latent and active abPPO4 mushroom tyrosinase cocrystallized with hexatungstotellurate(VI) in a single crystal. Acta Crystallographica Sect D. 2014;70(9):2301-15. doi:10.1107/S1399004714013777.

27. Mauracher SG, Molitor C, Al-Oweini R, Kortz U, Rompel A. Crystallization and preliminary $X$-ray crystallographic analysis of latent isoform PPO4 mushroom (Agaricus bisporus) tyrosinase. Acta Crystallographica Secti F Struct Biol Commun. 2014;70(Pt 2):263-6. doi:10.1107/ s2053230x14000582.

28. Wu J, Chen H, Gao J, Liu X, Cheng W, Ma X. Cloning, characterization and expression of two new polyphenol oxidase cDNAs from Agaricus bisporus. Biotechnol Lett. 2010;32(10):1439-47. doi:10.1007/s10529-010-0329-2.

29. Grigoriev IV, Nikitin R, Haridas S, Kuo A, Ohm R, Otillar R, et al. MycoCosm portal: gearing up for 1000 fungal genomes. Nucleic Acids Res. 2014;42(Database issue):D699-704. doi:10.1093/nar/gkt1183.

30. Berka RM, Grigoriev IV, Otillar R, Salamov A, Grimwood J, Reid I, et al. Comparative genomic analysis of the thermophilic biomass-degrading fungi Myceliophthora thermophila and Thielavia terrestris. Nat Biotechnol. 2011;29(10):922-7. doi:10.1038/nbt.1976.

31. Gasparetti C, Faccio G, Arvas M, Buchert J, Saloheimo M, Kruus K. Discovery of a new tyrosinase-like enzyme family lacking a C-terminally processed domain: production and characterization of an Aspergillus oryzae catechol oxidase. Appl Microbiol Biotechnol. 2010;86(1):213-26. doi:10.1007/s00253-009-2258-3.

32. Zhao Z, Liu H, Wang C, Xu J-R. Comparative analysis of fungal genomes reveals different plant cell wall degrading capacity in fungi. BMC Genom. 2013;14(1):1-15. doi:10.1186/1471-2164-14-274.

33. Selinheimo E, NiEidhin D, Steffensen C, Nielsen J, Lomascolo A, Halaouli S, et al. Comparison of the characteristics of fungal and plant tyrosinases. J Biotechnol. 2007;130(4):471-80. doi:10.1016/j.jbiotec.2007.05.018.

34. Zhou P, Smith NL, Lee CY. Potential purification and some properties of Monroe apple peel polyphenol oxidase. J Agric Food Chem. 1993;41(4):532-6. doi:10.1021/jf00028a004.

35. Paul B, Gowda LR. Purification and characterization of a polyphenol oxidase from the seeds of field bean (Dolichos lablab). J Agric Food Chem. 2000;48(9):3839-46. doi:10.1021/jf000296s.

36. Zou Y, Hu W, Jiang A, Ma K. Partial purification and characterization of a novel extracellular tyrosinase from Auricularia auricula. Appl Biochem Biotechnol. 2013;172(3):1460-9. doi:10.1007/s12010-013-0638-8.

37. Bissaro B, Rohr AK, Skaugen M, Forsberg Z, Horn SJ, Vaaje-Kolstad G, et al. Fenton-type chemistry by a copper enzyme: molecular mechanism of polysaccharide oxidative cleavage. bioRxiv. 2016. doi:10.1101/097022.

38. Rosazza JPN, Huang Z, Dostal L, Volm T, Rousseau B. Review: biocatalytic transformations of ferulic acid: an abundant aromatic natural product. J Ind Microbiol. 1995;15(6):457-71. doi:10.1007/bf01570016.

39. Schweigert N, Zehnder AJB, Eggen RIL. Chemical properties of catechols and their molecular modes of toxic action in cells, from microorganisms to mammals. Environ Microbiol. 2001;3(2):81-91. doi:10.1046/j.1462-2920.2001.00176.x.
40. Raymond J, Rakariyatham N, Azanza JL. Purification and some properties of polyphenoloxidase from sunflower seeds. Phytochemistry. 1993;34(4):927-31. doi:10.1016/S0031-9422(00)90689-7.

41. Lee CY, Smith NL, Pennesi AP. Polyphenoloxidase from DeChaunac grapes. J Sci Food Agric. 1983;34(9):987-91. doi:10.1002/jsfa.2740340915.

42. Hakulinen N, Gasparetti C, Kaljunen H, Kruus K, Rouvinen J. The crystal structure of an extracellular catechol oxidase from the ascomycete fungus Aspergillus oryzae. JBIC, J Biol Inorg Chem. 2013;18(8):917-29. doi:10.1007/s00775-013-1038-9.

43. Solem E, Tuczek F, Decker H. Tyrosinase versus catechol oxidase: one asparagine makes the difference. Angewandte Chemie Int Edit. 2016;55(8):2884-8. doi:10.1002/anie.201508534.

44. Klabunde T, Eicken C, Sacchettini JC, Krebs B. Crystal structure of a plant catechol oxidase containing a dicopper center. Nat Struct Biol. 1998;5(12):1084-90. doi:10.1038/4193.

45. Ismaya WT, Rozeboom HJ, Weijn A, Mes JJ, Fusetti F, Wichers HJ, et al. Crystal structure of Agaricus bisporus mushroom tyrosinase: identity of the tetramer subunits and interaction with tropolone. Biochemistry. 2011;50(24):5477-86. doi:10.1021/bi200395t.

46. Decker $\mathrm{H}$, Schweikardt T, Tuczek F. The first crystal structure of tyrosinase: all questions answered? Angewandte Chemie Int Edit. 2006;45(28):454650. doi:10.1002/anie.200601255.

47. Marusek CM, Trobaugh NM, Flurkey WH, Inlow JK. Comparative analysis of polyphenol oxidase from plant and fungal species. J Inorg Biochem. 2006;100(1):108-23. doi:10.1016/j.jinorgbio.2005.10.008.

48. Strothkamp KG, Jolley RL, Mason HS. Quaternary structure of mushroom tyrosinase. Biochem Biophys Res Commun. 1976;70(2):519-24. doi:10.1016/0006-291X(76)91077-9.

49. Kubicek CP, Kubicek EM. Enzymatic deconstruction of plant biomass by fungal enzymes. Curr Opin Chem Biol. 2016;35:51-7. doi:10.1016/j. cbpa.2016.08.028.

50. Goldfeder M, Kanteev M, Adir N, Fishman A. Influencing the monophenolase/diphenolase activity ratio in tyrosinase. Biochimica et Biophysica Acta Proteins Proteom. 2013;1834(3):629-33. doi:10.1016/j. bbapap.2012.12.021.

51. Visser H, Joosten V, Punt PJ, Gusakov AV, Olson PT, Joosten R, et al. Development of a mature fungal technology and production platform for industrial enzymes based on a Myceliophthora thermophila isolate, previously known as Chrysosporium lucknowense C1. Ind Biotechnol. 2011;7:214-23. doi:10.1089/ind.2011.0003.

52. Punt PJ, Burlingame RP, Pynnonen CM, Olson PT, Wery J, Visser J, Heinrich, et al. Chrysosporium lucknowense protein production system. Patent WO/2010/107303; 2010 .

53. Zhang YHP, Cui J, Lynd LR, Kuang LR. A transition from cellulose swelling to cellulose dissolution by o-phosphoric acid: evidence from enzymatic hydrolysis and supramolecular structure. Biomacromol. 2006;7(2):644-8. doi:10.1021/bm050799c.

54. Camacho C, Coulouris G, Avagyan V, Ma N, Papadopoulos J, Bealer K, et al. BLAST + : architecture and applications. BMC Bioinform. 2009;10(1):1-9. doi:10.1186/1471-2105-10-421.

55. Wichers HJ, Recourt K, Hendriks M, Ebbelaar CEM, Biancone G, Hoeberichts FA, et al. Cloning, expression and characterisation of two tyrosinase cDNAs from Agaricus bisporus. Appl Microbiol Biotechnol. 2003;61(4):33641. doi:10.1007/s00253-002-1194-2.

56. N-y Li, W-m Cai, Q-I Jin, Q-p Qin, F-I Ran. Molecular cloning and expression of polyphenoloxidase genes from the mushroom, Agaricus bisporus. Agric Sci China. 2011;10(2):185-94. doi:10.1016/S1671-2927(09)60305-9.

57. Kubicek CP, Starr TL, Glass NL. Plant cell wall-degrading enzymes and their secretion in plant-pathogenic fungi. Ann Rev Phytopathol. 2014;52(1):427-51. doi:10.1146/annurev-phyto-102313-045831.

58. Team RC. R: a language and environment for statistical computing. Vienna: R Foundation for Statistical Computing; 2016. https://www.Rproject.org. 